\title{
Monomorphic ESS does not imply the stability of the corresponding polymorphic state in the replicator dynamics in matrix games under time constraints
}

\author{
Tamás Varga* \\ Bolyai Institute \\ University of Szeged \\ Aradi vértanúk tere 1., Szeged 6720, Hungary \\ vargata@math.u-szeged.hu \\ József Garay \\ Centre for Ecological Research, Institute of Evolution \\ Konkoly-Thege M. út 29-33., Budapest H-1121, Hungary \\ garayj@caesar.elte.hu
}

\begin{abstract}
One of the main result in the theory of classical evolutionary matrix games (Maynard Smith and Price 1973, Maynard Smith 1982) claims that monomorphic ESS condition implies the stability of the corresponding state of the polymorphic replicator dynamics (Hofbauer et al. 1979, Zeeman 1980). The picture was then refined by Cressman (1990) introducing the strong stability concept which says that if there is a monomorphic ESS then stable polymorphism is established in polymorphic populations. In this paper we demonstrate with examples that this relationship generally does not hold in three or higher dimensions if times related to the interactions vary with the strategies of the participants.
\end{abstract}

Keywords: evolutionary game theory; time constraints; monomorphic ESS; replicator dynamics; counterexample

\section{Introduction}

The classical evolutionary game theoretical models concentrate on the frequency dependent selection situation, when the fitness of existing phenotypes depend on the average behavior of the whole population (Maynard Smith and Price 1973, Maynard Smith 1982, Chapter 6-7 in Hofbauer and Sigmund 1998, Chapter 6 in Broom and Rychtář 2013). Two basic selection situations are distinguished in this theory: The first one is the monomorphic situation, when the overwhelming majority of the population has the same resident phenotype (e.g. mixed of $N$ pure strategy, or pure), and only a small part of the population is formed by one type of mutant phenotype. For the monomorphic selectionary situation Maynard Smith introduced the following verbal static definition: "An ESS is a strategy such that, if all the members of a population adopt it, then no mutant strategy could invade the population under the influence of natural selection" (Maynard Smith 1982, p. 10). Observe that this static ESS concept

\footnotetext{
${ }^{*}$ Author for correspondence
} 
relies implicitly upon an underlying dynamic (for example, see Chapter 7.3 in Hofbauer and Sigmund 1998), since for each mutant, the relative frequencies of the monomorphic ESS increases from generation to generation, so the ESS is always fixed by the natural selection, when the mutation is rare.

The second selection situation is the polymorphic one, when in the population there are $N$ different genetically fixed pure phenotypes. In the frequency dependent matrix games, a locally asymptotically stable rest point of the replicator dynamics can be considered to be the endpoint of the evolution (Taylor and Yonker 1978, Hofbauer and Sigmund 1998).

It is well-known that the state corresponding to a monomorphic ESS is a locally asymptotically stable rest point of the replicator dynamics (Taylor and Yonker 1978, Hofbauer et al. 1979, Zeeman 1980). The converse is not true generally (see e.g. the matrix (7.12) on p. 71 in Hofbauer and Sigmund 1998). The relationship holds not only for the matrix games but for some cases when the fitness function is not bilinear in the phenotype of the focal individual and the population average strategy (indeed, it is enough to assume the linearity of the fitness function in the strategy of the focal individual, see e.g. Thomas (1985), Hofbauer and Sigmund 1998 Section 22 and Cressman et al. 2003). However, the validity of the folk theorem is an open question for other fitness functions.

The precise characterization of the ESS from dynamical aspects is given by the so called strong stability concept (Cressman 1992) also introduced in the framework of classical replicator dynamics. Consider a population of phenotypes which can realize the ESS as a convex combination. A strategy is an ESS if and only if the average strategy of any population of phenotypes which can mix the ESS tends to the ESS under the replicator dynamics. The validity of this result is also an open question for fitness functions which are not linear in the fitness of the focal individual.

Recently, a special class of evolutionary games has been considered, namely, the matrix games under time constraints (see details in Section 2) in which the consequence of an interaction is not only an intake but a waiting time. As a consequence of the latter, the fitness function is generally neither linear in the strategy of the focal individual nor in the average strategy of the population any longer. It was proved that the corresponding state of an ESS with respect to a matrix game under time constraints is asymptotically stable rest point of the replicator dynamics with respect to strategies the convex combinations of which form a one-dimensional manifold containing the ESS (Corollary 4.3 in Garay et al. 2018, Theorem 3.3 and 3.4 in Varga et al. 2020). This also means that the characterization of ESS for matrix games under time constraints by strong stability holds for two-dimensional strategies. However, the proofs exploit that the set of the convex combinations of the strategies for which the replicator dynamics is considered is one-dimensional. The higher dimensional case remained open.

In this paper, we give examples for matrix games under time constraints which show that the evolutionary stability of a strategy does generally not imply the local asymptotic stability of the corresponding state of the replicator dynamics even for 3 dimensional strategies.

\section{Preliminaries}

We introduce some functions and notations necessary to our calculations (see Garay et al. 2017, Garay et al. 2018 for the detailed backgrounds of these expressions). Let $S_{N}$ denote the set $\left\{\mathbf{q}=\left(q_{1}, q_{2}, \ldots, q_{N}\right) \in \mathbb{R}^{N}: q_{1}+q_{2}+\cdots+q_{N}=1\right.$ and $\left.q_{1}, q_{2}, \ldots, q_{N} \geq 0\right\}$ ( $N$ is a positive integer). The elements of $S_{N}$ are called strategies or (pheno)types. The special strategies $\mathbf{e}_{1}=(1,0, \ldots, 0), \mathbf{e}_{2}=(0,1,0, \ldots, 0), \ldots, \mathbf{e}_{N-1}=(0,0, \ldots, 0,1,0), \mathbf{e}_{N}=(0,0, \ldots, 0,1)$ 
are called the pure strategies. An individual following strategy $\mathbf{q}$ is called $\mathbf{q}$ individual or q-strategist. If an $\mathbf{e}_{i}$ individual interacts with an $\mathbf{e}_{j}$ individual then the payoff of the $\mathbf{e}_{i}$ individual is $a_{i j}$ while the $N \times N$ matrix $A=\left(a_{i j}\right)$ is called the payoff matrix. Similarly, $\tau_{i j}$ denotes the waiting time the $\mathbf{e}_{i}$ individual should wait after such an interaction before searching for a new opponent and the $N \times N$ matrix $T=\left(\tau_{i j}\right)$ is called the time-constraint matrix. It is assumed that $\tau_{i j} \geq 0$. If $\mathbf{q} \in S_{N}$ is a strategy then $\rho(\mathbf{q})$ denotes the unique solution in $[0,1]$ to the equation

$$
\rho=\frac{1}{1+\mathbf{q} T \rho \mathbf{q}}
$$

that is,

$$
\rho(\mathbf{q})=\frac{-1+\sqrt{1+4 \mathbf{q} T \mathbf{q}}}{2 \mathbf{q} T \mathbf{q}}=\frac{2}{1+\sqrt{1+4 \mathbf{q} T \mathbf{q}}}
$$

(Lemma 2 in Garay et al. 2017). Then

$$
\rho_{\mathbf{p}}(\mathbf{q}):=\frac{1}{1+\mathbf{p} T \rho(\mathbf{q}) \mathbf{q}},
$$

$\omega(\mathbf{q}):=\frac{\mathbf{q} A \rho(\mathbf{q}) \mathbf{q}}{1+\mathbf{q} T \rho(\mathbf{q}) \mathbf{q}}=\rho(\mathbf{q}) \mathbf{q} A \rho(\mathbf{q}) \mathbf{q} \quad$ and $\quad \omega_{\mathbf{p}}(\mathbf{q}):=\frac{\mathbf{p} A \rho(\mathbf{q}) \mathbf{q}}{1+\mathbf{p} T \rho(\mathbf{q}) \mathbf{q}}=\rho_{\mathbf{p}}(\mathbf{q}) \mathbf{p} A \rho(\mathbf{q}) \mathbf{q}$

(If a strategy $\mathbf{q} \in S_{N}$ is multiplied by an $N \times N$ matrix $\mathrm{M}$ from the right then $\mathbf{q}$ should be considered a row vector while if it is multiplied by the matrix $M$ from the left then $q$ should be considered a column vector.) It follows that both $\rho(\mathbf{q})$ and $\rho_{\mathbf{p}}(\mathbf{q})$ has continuous second order partial derivatives with respect to the coordinates of $\mathbf{q}=\left(q_{1}, q_{2}, \ldots, q_{N}\right) \in S_{N}$. $\rho(\mathbf{q})$ can be interpreted as the proportion of the active individuals in a population of individuals following strategy q. $\omega(\mathbf{q})$ represents the fitness of an individual in this population while $\omega_{\mathbf{p}}(\mathbf{q})$ can be considered as the theoretical fitness of one and only individual of strategy $\mathbf{p}$ in a large population of $\mathbf{q}$ individuals (as if a single $\mathbf{p}$-strategist were in an infinitely large population of individuals of phenotype $\mathbf{q}$ where $\rho_{\mathbf{p}}$ is the probability that the $\mathbf{p}$ individual is active in a given moment).

If $\mathbf{p}, \mathbf{q} \in S_{N}$ and $\varepsilon \in[0,1]$ let $\rho_{\mathbf{p}}(\mathbf{p}, \mathbf{q}, \varepsilon)$ and $\rho_{\mathbf{q}}(\mathbf{p}, \mathbf{q}, \varepsilon)$, respectively, be the unique solution in $[0,1]$ to the equation system

$$
\begin{aligned}
\rho_{\mathbf{p}} & =\frac{1}{1+\mathbf{p} T\left[(1-\varepsilon) \rho_{\mathbf{p}} \mathbf{p}+\varepsilon \rho_{\mathbf{q}} \mathbf{q}\right]} \\
\rho_{\mathbf{q}} & =\frac{1}{1+\mathbf{q} T\left[(1-\varepsilon) \rho_{\mathbf{p}} \mathbf{p}+\varepsilon \rho_{\mathbf{q}} \mathbf{q}\right]} .
\end{aligned}
$$

(Lemma 2 in Garay et al. 2017). $\rho_{\mathbf{p}}$ and $\rho_{\mathbf{q}}$, respectively, are the proportion of active individuals in the subpopulation of phenotype $\mathbf{p}$ and phenotype $\mathbf{q}$, respectively, in a population where $(1-\varepsilon)$ is the frequency of $\mathbf{p}$ individuals and $\varepsilon$ that of $\mathbf{q}$ individuals. The fitness of these types is described as follows

$$
\begin{aligned}
& \omega_{\mathbf{p}}(\mathbf{p}, \mathbf{q}, \varepsilon)=\frac{\mathbf{p} A\left[(1-\varepsilon) \rho_{\mathbf{p}} \mathbf{p}+\varepsilon \rho_{\mathbf{q}} \mathbf{q}\right]}{1+\mathbf{p} T\left[(1-\varepsilon) \rho_{\mathbf{p}} \mathbf{p}+\varepsilon \rho_{\mathbf{q}} \mathbf{q}\right]}=\rho_{\mathbf{p}} \mathbf{p} A\left[(1-\varepsilon) \rho_{\mathbf{p}} \mathbf{p}+\varepsilon \rho_{\mathbf{q}} \mathbf{q}\right] \\
& \omega_{\mathbf{q}}(\mathbf{p}, \mathbf{q}, \varepsilon)=\frac{\mathbf{q} A\left[(1-\varepsilon) \rho_{\mathbf{p}} \mathbf{p}+\varepsilon \rho_{\mathbf{q}} \mathbf{q}\right]}{1+\mathbf{q} T\left[(1-\varepsilon) \rho_{\mathbf{p}} \mathbf{p}+\varepsilon \rho_{\mathbf{q}} \mathbf{q}\right]}=\rho_{\mathbf{q}} \mathbf{q} A\left[(1-\varepsilon) \rho_{\mathbf{p}} \mathbf{p}+\varepsilon \rho_{\mathbf{q}} \mathbf{q}\right] .
\end{aligned}
$$

Note that $\rho(\mathbf{q})=\rho_{\mathbf{q}}(\mathbf{q}, \mathbf{p}, 0)=\rho_{\mathbf{q}}(\mathbf{p}, \mathbf{q}, 1), \omega(\mathbf{q})=\omega_{\mathbf{q}}(\mathbf{q})=\omega_{\mathbf{q}}(\mathbf{q}, \mathbf{p}, 0)=\omega_{\mathbf{q}}(\mathbf{p}, \mathbf{q}, 1), \rho_{\mathbf{p}}(\mathbf{q})=$ $\rho_{\mathbf{p}}(\mathbf{p}, \mathbf{q}, 1)=\rho_{\mathbf{p}}(\mathbf{q}, \mathbf{p}, 0)$ and $\omega_{\mathbf{p}}(\mathbf{q})=\omega_{\mathbf{p}}(\mathbf{p}, \mathbf{q}, 1)=\omega_{\mathbf{p}}(\mathbf{q}, \mathbf{p}, 0)$ for any $\mathbf{p}, \mathbf{q} \in S_{N}$ and $\varepsilon \in$ 
$[0,1]$. Also, if $\mathbf{x} \in S_{n}$ ( $n$ is a positive integer which can differ from $\left.N\right)$ and $\mathbf{q}_{1}, \ldots, \mathbf{q}_{n} \in S_{N}$ are fixed strategies then let $\varrho_{i}(\mathbf{x})$ be the unique solution in $[0,1]$ to the equation system:

$$
\varrho_{i}=\frac{1}{1+\mathbf{q}_{i} T\left[x_{1} \varrho_{1} \mathbf{q}_{1}+x_{2} \varrho_{2} \mathbf{q}_{2}+\cdots+x_{n} \varrho_{n} \mathbf{q}_{n}\right]} \quad(i=1,2, \ldots, n)
$$

(Lemma 2 in Garay et al. 2017). Then

$$
\begin{aligned}
& \bar{\varrho}(\mathbf{x}):=x_{1} \varrho_{1}(\mathbf{x})+x_{2} \varrho_{2}(\mathbf{x})+\cdots+x_{n} \varrho_{n}(\mathbf{x}), \\
& \overline{\mathbf{h}}(\mathbf{x}):=x_{1} \frac{\varrho_{1}(\mathbf{x})}{\bar{\varrho}(\mathbf{x})} \mathbf{q}_{1}+x_{2} \frac{\varrho_{2}(\mathbf{x})}{\bar{\varrho}(\mathbf{x})} \mathbf{q}_{2}+\cdots+x_{n} \frac{\varrho_{n}(\mathbf{x})}{\varrho(\mathbf{x})} \mathbf{q}_{n}, \\
& W_{i}(\mathbf{x}):=\frac{\mathbf{q}_{i} A \sum_{j=1}^{n} x_{j} \varrho_{j}(\mathbf{x}) \mathbf{q}_{j}}{1+\mathbf{q}_{i} T \sum_{j=1}^{n} x_{j} \varrho_{j}(\mathbf{x}) \mathbf{q}_{j}}=\varrho_{i}(\mathbf{x}) \mathbf{q}_{i} A \varrho(\mathbf{x}) \overline{\mathbf{h}}(\mathbf{x}) \quad \text { and } \\
& \bar{W}(\mathbf{x}):=x_{1} W_{1}(\mathbf{x})+x_{2} W_{2}(\mathbf{x})+\cdots+x_{n} W_{n}(\mathbf{x})=\bar{\varrho}(\mathbf{x}) \overline{\mathbf{h}}(\mathbf{x}) A \bar{\varrho}(\mathbf{x}) \overline{\mathbf{h}}(\mathbf{x}) .
\end{aligned}
$$

Here $\varrho_{i}$ is the proportion of active individuals in the subpopulation of $\mathbf{q}_{i}$ individuals. $\bar{\varrho}$ gives the proportion of active individuals in the whole population where $x_{i}$ is the frequency of type $\mathbf{q}_{i} . \overline{\mathbf{h}}(\mathbf{x})$ can be considered as the average strategy of the active individuals. $W_{i}$ describes the fitness of an individual following strategy $\mathbf{q}_{i}$ while $\bar{W}$ is the average fitness. Note that if the entries of $T$ are all equal we get back the case of classical evolutionary matrix games (Maynard Smith 1982, Chapter 6-7 in Hofbauer and Sigmund 1998).

We quote some statements crucial in understanding the relationship between the monomorphic and the polymorphic model.

Lemma 2.1 (Garay et al. 2018, Proposition 3.1) Consider a polymorphic population of phenotypes $\mathbf{q}_{1}, \mathbf{q}_{2}, \ldots, \mathbf{q}_{n}$ with frequency distribution $\left(x_{1}, x_{2}, \ldots, x_{n}\right)$. Then $\bar{\varrho}(\mathbf{x})=\rho(\overline{\mathbf{h}}(\mathbf{x}))$ and $\omega(\overline{\mathbf{h}}(\mathbf{x}))=\bar{W}(\mathbf{x})$, that is the fitness of an $\overline{\mathbf{h}}(\mathbf{x})$ individual is just the average fitness $\bar{W}(\mathbf{x})$ of the phenotypes $\mathbf{q}_{1}, \mathbf{q}_{2}, \ldots, \mathbf{q}_{n}$ in the polymorphic model.

For classical matrix games, it is clear that if $\mathbf{p}=\left(p_{1}, p_{2}, \ldots, p_{N}\right)$ is a strategy then $\left(x_{1}, x_{2}, \ldots, x_{N}\right)$ with $x_{i}=p_{i}$ is the corresponding state in a polymorphic systems of pure phenotypes $\mathbf{e}_{1}, \mathbf{e}_{2}, \ldots, \mathbf{e}_{N}$ individuals in the sense that $\sum_{i} x_{i} \mathbf{e}_{i}=\mathbf{p}$. Consequently, if, for example, $\mathbf{p}$ is an ESS then it is natural to investigate the stability property of the state $\left(x_{1}, x_{2}, \ldots, x_{N}\right)$ with $x_{i}=p_{i}$ for the replicator dynamics with respect to $\mathbf{e}_{1}, \mathbf{e}_{2}, \ldots, \mathbf{e}_{N}$. If the replicator dynamics is considered for arbitrary strategies $\mathbf{q}_{1}, \mathbf{q}_{2}, \ldots, \mathbf{q}_{n}$ then it is also clear that the corresponding state(s) of $\mathbf{p}$ is (are) the state(s) $\left(x_{1}, x_{2}, \ldots, x_{n}\right)$ with $\mathbf{p}=x_{1} \mathbf{q}_{1}+x_{2} \mathbf{q}_{2}+\cdots+x_{n} \mathbf{q}_{n}$, that is, the state(s) at which the average strategy of the population is $\mathbf{p}$. For matrix games under time constraints, however, the situation is generally not so obvious. The answer can be found in the next lemma which says that the corresponding state is the state at which the average strategy of the active part of the population is $\mathbf{p}$.

Lemma 2.2 (Garay et al. 2018, Corollary 6.3) Consider a population of phenotypes $\mathbf{q}_{1}$, $\mathbf{q}_{2}, \ldots, \mathbf{q}_{n} \in S_{N}$. Assume that $\mathbf{r}=\theta_{1} \mathbf{q}_{1}+\theta_{2} \mathbf{q}_{2}+\cdots+\theta_{n} \mathbf{q}_{n}$ with some $\boldsymbol{\theta}=\left(\theta_{1}, \theta_{2}, \ldots, \theta_{n}\right) \in S_{n}$. If

$$
x_{i}=x_{i}(\boldsymbol{\theta})=\frac{\rho(\mathbf{r})}{\rho_{\mathbf{q}_{i}}(\mathbf{r})} \theta_{i} \quad(i=1,2, \ldots, n)
$$

then $\overline{\mathbf{h}}(\mathbf{x})=\mathbf{r}$ where $\mathbf{x}=\mathbf{x}(\boldsymbol{\theta})=\left(x_{1}(\boldsymbol{\theta}), x_{2}(\boldsymbol{\theta}), \ldots, x_{n}(\boldsymbol{\theta})\right)$, that is, the average strategy of active individuals in a population of $\mathbf{q}_{1}, \mathbf{q}_{2}, \ldots, \mathbf{q}_{n}$ individuals with frequencies $x_{1}(\boldsymbol{\theta})$, $x_{2}(\boldsymbol{\theta}), \ldots, x_{n}(\boldsymbol{\theta})$ is $\theta_{1} \mathbf{q}_{1}+\theta_{2} \mathbf{q}_{2}+\cdots+\theta_{n} \mathbf{q}_{n}=\mathbf{r}$. Moreover, $\mathbf{x}(\boldsymbol{\theta})$ is the unique state for which $\theta_{i}=x_{i} \varrho(\mathbf{x}) / \bar{\varrho}(\mathbf{x})(i=1,2, \ldots, n)$ holds. 
We consider two particular cases. One of them is when $n=N=3, \mathbf{q}_{1}=\mathbf{e}_{1}, \mathbf{q}_{2}=\mathbf{e}_{2}$ and $\mathbf{q}_{3}=\mathbf{e}_{3}$. Let $\mathbf{p}=\left(p_{1}, p_{2}, 1-p_{1}-p_{2}\right)=p_{1} \mathbf{e}_{1}+p_{2} \mathbf{e}_{2}+\left(1-p_{1}-p_{2}\right) \mathbf{e}_{3}$ be a strategy from $S_{3}$. By the previous lemma, there exits a unique ${ }^{1}$ state $\mathbf{x}(\mathbf{p}) \in S_{3}$ with $\overline{\mathbf{h}}(\mathbf{x}(\mathbf{p}))=\mathbf{p}$, namely, $x_{i}(\mathbf{p})=p_{i} \rho(\mathbf{p}) / \rho_{\mathbf{e}_{i}}(\mathbf{p})$. So $x_{i}(\mathbf{p})$ has continuous partial derivatives with respect to the variables $p_{1}$ and $p_{2}$. If it is necessary to emphasize that $\mathbf{x}(\mathbf{p})$ is concerned with this case then we use the notation $\mathbf{x}^{R}(\mathbf{p})$ ("R" refers to "restricted").

The other case is when $N=3, n=4, \mathbf{q}_{1}=\mathbf{e}_{1}, \mathbf{q}_{2}=\mathbf{e}_{2}, \mathbf{q}_{3}=\mathbf{e}_{3}$ and $\mathbf{q}_{4}=\mathbf{p}^{*}:=$ $(1 / 3,1 / 3,1 / 3)$. Then most of the strategies from $S_{3}$, namely, the strategies in the interior of $S_{3}$ can be expressed in the form $\theta_{1} \mathbf{e}_{1}+\theta_{2} \mathbf{e}_{2}+\theta_{3} \mathbf{e}_{3}+\theta_{4} \mathbf{p}^{*}$ in more than one way. For such a strategy $\mathbf{q}$, there are thus more then one state $\mathbf{x}=\left(x_{1}, x_{2}, x_{3}, x_{4}\right)$ with $\overline{\mathbf{h}}(\mathbf{x})=\mathbf{q}$. So it would be ambiguous the notation $\mathbf{x}(\mathbf{q})$ for a state with $\overline{\mathbf{h}}(\mathbf{x}(\mathbf{q}))=\mathbf{q}$. Therefore, rather than associating a strategy $\mathbf{q}$ to such a state, we instead associate the coefficient-list $\boldsymbol{\theta}=\left(\theta_{1}, \theta_{2}, \theta_{3}, \theta_{4}\right)$ from the convex combination $\theta_{1} \mathbf{e}_{1}+\theta_{2} \mathbf{e}_{2}+\theta_{3} \mathbf{e}_{3}+\theta_{4} \mathbf{p}=\mathbf{q}$ to the state $\mathbf{x}=\mathbf{x}(\boldsymbol{\theta})=\mathbf{x}\left(\theta_{1}, \theta_{2}, \theta_{3}, \theta_{4}\right)$ with $x_{i}(\boldsymbol{\theta})=\theta_{i} \rho(\mathbf{q}) / \rho_{\mathbf{q}_{i}}(\mathbf{q})$. If it is necessary to emphasize that $\mathbf{x}(\boldsymbol{\theta})$ is concerned with this case then we use the notation $\mathbf{x}^{E}(\boldsymbol{\theta})$ ("E" refers to "extended"). Observe that if the vector $\left(x_{1}, x_{2}, 1-x_{1}-x_{3}, 0\right) \in S_{4}$ is identified with the vector $\left(x_{1}, x_{2}, 1-x_{1}-x_{3}\right) \in S_{3}$, then the map $\mathrm{x}^{R}$ is just the restriction of the map $\mathrm{x}^{E}$ to the set $S_{3}$.

Note the following "exchange rule" between the strategies and states. This is just the simple consequence of the preceding lemmas and the definitions of the expressions above.

Corollary 2.3 (Exchange rule) Let $\mathbf{p}$ be a strategy in $S_{3}$ and consider the polymorphic population of the pure phenotypes $\mathbf{e}_{1}, \mathbf{e}_{2}, \mathbf{e}_{3}$. Then the following relationships hold:

$$
\begin{aligned}
\overline{\mathbf{h}}(\mathbf{x}(\mathbf{p})) & =\mathbf{p}, & \mathbf{x}(\overline{\mathbf{h}}(\mathbf{x})) & =\mathbf{x}, \\
\varrho_{i}(\mathbf{x}(\mathbf{p})) & =\rho_{\mathbf{e}_{i}}(\mathbf{p}), & \rho_{\mathbf{e}_{i}}\left(\overline{\mathbf{h}}(\mathbf{x}), \mathbf{e}_{i}, 0\right) & =\rho_{\mathbf{e}_{i}}(\overline{\mathbf{h}}(\mathbf{x}))=\varrho_{i}(\mathbf{x}), \\
\bar{\varrho}(\mathbf{x}(\mathbf{p})) & =\rho(\mathbf{p}), & \rho(\overline{\mathbf{h}}(\mathbf{x})) & =\bar{\varrho}(\mathbf{x}), \\
W_{i}(\mathbf{x}) & =\omega_{\mathbf{e}_{i}}\left(\overline{\mathbf{h}}(\mathbf{x}), \mathbf{e}_{i}, 0\right)=\omega_{\mathbf{e}_{i}}(\overline{\mathbf{h}}(\mathbf{x})), & \omega_{\mathbf{e}_{i}}(\mathbf{p}) & =\omega_{\mathbf{e}_{i}}\left(\mathbf{p}, \mathbf{e}_{i}, 0\right)=W_{i}(\mathbf{x}(\mathbf{p})), \\
\bar{W}(\mathbf{x}) & =\omega(\overline{\mathbf{h}}(\mathbf{x})), & \omega(\mathbf{p}) & =\bar{W}(\mathbf{x}(\mathbf{p})) .
\end{aligned}
$$

Using the notations above the evolutionary stability of a strategy is defined as follows.

Definition 2.4 A strategy $\mathbf{p}^{*}$ is uniformly evolutionary stable strategy of the matrix game under time constraints (UESS in short) if there is an $\varepsilon_{0}>0$ such that

$$
\omega_{\mathbf{p}^{*}}\left(\mathbf{p}^{*}, \mathbf{q}, \varepsilon\right)>\omega_{\mathbf{q}}\left(\mathbf{p}^{*}, \mathbf{q}, \varepsilon\right)
$$

for any strategy $\mathbf{q} \neq \mathbf{p}^{*}$.

The adverb "uniformly" is used because $\varepsilon_{0}$ is not depend on $\mathbf{q}$. If $\varepsilon_{0}$ can depend on $\mathbf{q}$ then $\mathbf{p}^{*}$ is called ESS without the adverb "uniformly". Clearly, if $\mathbf{p}^{*}$ is a UESS then $\mathbf{p}^{*}$ is a ESS too. The converse is true in the case of the classical evolutionary matrix games (without time constraints) (Theorem 6.4.1 in Hofbauer and Sigmund 1998) but it is an open question for all matrix games under time constraints. We do not want to deal with this problem in this article only we wanted to explain the cause of using the term "UESS". Incidentally, this distinction between UESS and ESS naturally emerges for non-linear fitness functions (Bomze and Weibull 1995) and UESS is more appropriate for those (Pohley and Thomas 1983).

We look for examples having a UESS $\mathbf{p}^{*}$ such that the corresponding state $\mathbf{x}\left(\mathbf{p}^{*}\right)$ is unstable rest point of the replicator dynamics with respect to the pure strategies. To find such a UESS we use the following characterization which make the calculation easier.

\footnotetext{
${ }^{1}$ The uniqueness trivially follows from the fact that every $\mathbf{p}=\left(p_{1}, p_{2}, p_{3}\right) \in S_{3}$ is a unique convex combination of the pure strategies: $\mathbf{p}=p_{1} \mathbf{e}_{1}+p_{2} \mathbf{e}_{2}+p_{3} \mathbf{e}_{3}$.
} 
Theorem 2.5 (Corollary 3.2 in Varga et al. 2020) A strategy $\mathrm{p}^{*}$ is a UESS if and only if there is a $\delta>0$ such that $\underbrace{\omega_{\mathbf{p}^{*}}\left(\mathbf{p}^{*}, \mathbf{q}, 1\right)}_{=\omega_{\mathbf{p}^{*}}(\mathbf{q})}>\underbrace{\omega_{\mathbf{q}}\left(\mathbf{p}^{*}, \mathbf{q}, 1\right)}_{=\omega_{\mathbf{q}}(\mathbf{q})}$ whenever $0<\left\|\mathbf{p}^{*}-\mathbf{q}\right\|<\delta$.

Accordingly, if

$$
f\left(q_{1}, q_{2}\right)=f(\mathbf{q})=f_{\mathbf{p}^{*}}(\mathbf{q})=\omega_{\mathbf{p}^{*}}\left(\mathbf{p}^{*}, \mathbf{q}, 1\right)-\omega_{\mathbf{q}}\left(\mathbf{p}^{*}, \mathbf{q}, 1\right)=\omega_{\mathbf{p}^{*}}(\mathbf{q})-\omega(\mathbf{q}),
$$

then $\mathbf{p}$ is a UESS if and only if it is a strict local minimum of $f$. Note that since $f$ is defined on $S_{3}$ which is a 2 dimensional manifold in $\mathbb{R}^{3}$ it can be considered as a two variable function of $q_{1}, q_{2}$. It is well-known from multivariable calculus that if

[C1] $f_{q_{1}}^{\prime}\left(p_{1}, p_{2}\right)=f_{q_{2}}^{\prime}\left(p_{1}, p_{2}\right)=0$,

$[\mathrm{C} 2] f_{q_{1} q_{1}}^{\prime \prime}\left(p_{1}, p_{2}\right) f_{q_{2} q_{2}}^{\prime \prime}\left(p_{1}, p_{2}\right)>\left(f_{q_{1} q_{2}}^{\prime \prime}\left(p_{1}, p_{2}\right)\right)^{2}$ and

[C3] $f_{q_{1} q_{1}}^{\prime \prime}\left(p_{1}, p_{2}\right)>0$

then $\mathbf{p}=\left(p_{1}, p_{2}, 1-p_{1}-p_{2}\right)$ is a strict local minimum of $f$. (see e.g. Theorem 11 of Chapter 14, p. 838 in Thomas 2014). Consequently, if [C1], [C2], [C3] hold for $\mathbf{p}^{*}=(1 / 3,1 / 3,1 / 3)$ with respect to the matrices $A$ and $T$, furthermore, the state $\mathbf{x}\left(\mathbf{p}^{*}\right)$ corresponding to $\mathbf{p}^{*}$ is an unstable rest point of the replicator dynamics then we have found a counterexample.

Consider, therefore, the replicator dynamics for the polymorphic population consisting of the pure phenotypes $\mathbf{q}_{1}=\mathbf{e}_{1}, \mathbf{q}_{2}=\mathbf{e}_{2}, \mathbf{q}_{3}=\mathbf{e}_{3}$ with frequencies $x_{1}, x_{2}$ and $x_{3}=1-x_{1}-x_{2}$, respectively:

$$
\begin{aligned}
& \dot{x}_{1}=x_{1}\left[W_{1}(\mathbf{x})-\bar{W}(\mathbf{x})\right] \\
& \dot{x}_{2}=x_{2}\left[W_{2}(\mathbf{x})-\bar{W}(\mathbf{x})\right] \\
& \dot{x}_{3}=x_{3}\left[W_{3}(\mathbf{x})-\bar{W}(\mathbf{x})\right],
\end{aligned}
$$

where $W_{i}$ and $\bar{W}$ is defined in (2.3). Since $x_{1}+x_{2}+x_{3}=1$ also holds any two equations of the system determine the dynamics. In what follows, it will thus be sufficient to investigate the differential equation system

$$
\begin{aligned}
& \dot{x}_{1}=x_{1}\left[W_{1}(\mathbf{x})-\bar{W}(\mathbf{x})\right] \\
& \dot{x}_{2}=x_{2}\left[W_{2}(\mathbf{x})-\bar{W}(\mathbf{x})\right] .
\end{aligned}
$$

If $\mathbf{p}$ is a $\mathrm{NE}^{2}$, then the corresponding state $\mathbf{x}(\mathbf{p})$ is a rest point of the replicator dynamics (Lemma 3.2, Garay et al. 2018). Since $(1 / 3,1 / 3,1 / 3)$ is a UESS, which implies that it is a NE too (see the paragraph before Definition 2.2 in Garay et al. 2017), it follows that $\mathbf{x}(1 / 3,1 / 3,1 / 3)$ is a rest point of the replicator dynamics.

We would like that $\mathbf{x}(1 / 3,1 / 3,1 / 3)$ were unstable. This holds if the Jacobian matrix of the right hand side of (2.7) has a positive eigenvalue at $\mathbf{x}(1 / 3,1 / 3,1 / 3)$. It can be easily checked for a function of two variables that if the characteristic polynomials of the Jacobian matrix is $\lambda^{2}+b \lambda+c$ (where $\lambda$ is the variable), then the Jacobian matrix has a positive eigenvalue if and only if

[C4] $b<0$ or $c<0$

\footnotetext{
${ }^{2}$ A strategy $\mathbf{p}^{*}$ is called Nash equilibrium for the matrix game under time constraints (NE in short) if the inequality $\omega_{\mathbf{p}^{*}}\left(\mathbf{p}^{*}\right) \geq \omega_{\mathbf{q}}\left(\mathbf{p}^{*}\right)$ holds for any strategy $\mathbf{q} \in S_{N}$ (Definition 2.2 in Garay et al. 2018).
} 
(see, for example Chapter 4.3 in Kong 2014).

Conditions [C1]-[C4] mean equations and inequalities. Solving them one can find appropriate matrices $A$ and $T$ for which there is a UESS $\mathbf{p}$ but the corresponding state $\mathbf{x}(\mathbf{p})$ is unstable with respect to the replicator dynamics. Here we give three of such examples and check the conditions. We emphasize that the results concerning the local properties of equlibrium points and the global behaviour on the edges are exact while the results concerning the global behaviour in the interior of the simplex are only based on the simulated phase portrait.

The explicit calculation of $\varrho_{i}(i=1,2,3)$ is generally not possible but, for a given strategy $\mathbf{p}$, we can calculate the eigenvalues of the Jacobian matrix at the corresponding state $\mathbf{x}(\mathbf{p})$. For this purpose, applying the "exchange rule", rewrite (2.7) as follows

$$
\begin{aligned}
& \dot{x}_{1}=\bar{h}_{1}(\mathbf{x}) \frac{\rho(\overline{\mathbf{h}}(\mathbf{x}))}{\rho_{\mathbf{e}_{1}}(\overline{\mathbf{h}}(\mathbf{x}))}\left[\omega_{1}(\overline{\mathbf{h}}(\mathbf{x}))-\omega(\overline{\mathbf{h}}(\mathbf{x}))\right] \\
& \dot{x}_{2}=\bar{h}_{2}(\mathbf{x}) \frac{\rho(\overline{\mathbf{h}}(\mathbf{x}))}{\rho_{\mathbf{e}_{2}}(\overline{\mathbf{h}}(\mathbf{x}))}\left[\omega_{2}(\overline{\mathbf{h}}(\mathbf{x}))-\omega(\overline{\mathbf{h}}(\mathbf{x}))\right],
\end{aligned}
$$

where $\bar{h}_{1}, \bar{h}_{2}$ is just the first two components of $\overline{\mathbf{h}}$, that is, $\overline{\mathbf{h}}(\mathbf{x})=\left(\bar{h}_{1}(\mathbf{x}), \bar{h}_{2}(\mathbf{x}), 1-\bar{h}_{1}(\mathbf{x})-\right.$ $\left.\bar{h}_{2}(\mathbf{x})\right)$. If

$$
\begin{aligned}
& R_{1}\left(q_{1}, q_{2}\right)=q_{1} \frac{\rho(\mathbf{q})}{\rho_{\mathbf{e}_{1}}(\mathbf{q})}\left[\omega_{1}(\mathbf{q})-\omega(\mathbf{q})\right] \\
& R_{2}\left(q_{1}, q_{2}\right)=q_{2} \frac{\rho(\mathbf{q})}{\rho_{\mathbf{e}_{2}}(\mathbf{q})}\left[\omega_{2}(\mathbf{q})-\omega(\mathbf{q})\right],
\end{aligned}
$$

then the Jacobian matrix of the right hand side of (2.8) takes the following shape:

$$
\left(\begin{array}{cc}
\partial_{q_{1}} R_{1}\left(\bar{h}_{1}(\mathbf{x}), \bar{h}_{2}(\mathbf{x})\right) & \partial_{q_{2}} R_{1}\left(\bar{h}_{1}(\mathbf{x}), \bar{h}_{2}(\mathbf{x})\right) \\
\partial_{q_{1}} R_{2}\left(\bar{h}_{1}(\mathbf{x}), \bar{h}_{2}(\mathbf{x})\right) & \partial_{q_{2}} R_{2}\left(\bar{h}_{1}(\mathbf{x}), \bar{h}_{2}(\mathbf{x})\right)
\end{array}\right)\left(\begin{array}{cc}
\partial_{x_{1}} \bar{h}_{1}(\mathbf{x}) & \partial_{x_{2}} \bar{h}_{1}(\mathbf{x}) \\
\partial_{x_{1}} \bar{h}_{2}(\mathbf{x}) & \partial_{x_{2}} \bar{h}_{2}(\mathbf{x})
\end{array}\right)
$$

We need the Jacobian at state $\mathbf{x}(1 / 3,1 / 3,1 / 3)$. We therefore express $\mathbf{x}(\mathbf{q})$ as a function of $\mathbf{q}$ and then we apply the well-known inverse function theorem (see e.g. Theorem 9.22 and (49) in Chapter 9 (p. 181) in Rudin 1953) to the function $\overline{\mathbf{h}}(\mathbf{x})$. We obtain that

$$
\begin{aligned}
& \left(\begin{array}{ll}
\partial_{x_{1}} \bar{h}_{1}(\mathbf{x}) & \partial_{x_{2}} \bar{h}_{1}(\mathbf{x}) \\
\partial_{x_{1}} \bar{h}_{2}(\mathbf{x}) & \partial_{x_{2}} \bar{h}_{2}(\mathbf{x})
\end{array}\right)_{\mathbf{x}=\mathbf{x}(1 / 3,1 / 3,1 / 3)}=\left[\left(\begin{array}{cc}
\partial_{q_{1}} x_{1}(\mathbf{q}) & \partial_{q_{2}} x_{1}(\mathbf{q}) \\
\partial_{q_{1}} x_{2}(\mathbf{q}) & \partial_{q_{2}} x_{2}(\mathbf{q})
\end{array}\right)_{\mathbf{q}=(1 / 3,1 / 3,1 / 3)}\right]^{-1} \\
& =\frac{\left(\begin{array}{cc}
\partial_{q_{2}} x_{2}(\mathbf{q}) & -\partial_{q_{2}} x_{1}(\mathbf{q}) \\
-\partial_{q_{1}} x_{2}(\mathbf{q}) & \partial_{q_{1}} x_{1}(\mathbf{q})
\end{array}\right)_{\mathbf{q}=(1 / 3,1 / 3,1 / 3)}}{\operatorname{det}\left[\left(\begin{array}{cc}
\partial_{q_{1}} x_{1}(\mathbf{q}) & \partial_{q_{2}} x_{1}(\mathbf{q}) \\
\partial_{q_{1}} x_{2}(\mathbf{q}) & \partial_{q_{2}} x_{2}(\mathbf{q})
\end{array}\right)_{\mathbf{q}=(1 / 3,1 / 3,1 / 3)}\right.},
\end{aligned}
$$

where, of course, the determinant in the denominator of the rightmost side should differ from zero.

\section{Examples}

We are ready to present three examples for matrix games under time constraints for which there exist a UESS but the state corresponding to the UESS is an unstable rest point of 
the replicator dynamics with respect to the pure strategies. However, the global behaviour of the examples are distinct. The strategy space is $S_{3}=\left\{\left(q_{1}, q_{2}, q_{3}\right) \in \mathbb{R}^{3}: q_{i} \geq 0, i=\right.$ $1,2,3$, and $\left.q_{1}+q_{2}+q_{3}=1\right\}$. In each example, strategy $\mathbf{p}^{*}=(1 / 3,1 / 3,1 / 3)$ is a UESS. The corresponding state is $\mathbf{x}=\mathbf{x}\left(\mathbf{p}^{*}\right)=\mathbf{x}^{R}\left(\mathbf{p}^{*}\right)=\left(x_{1}\left(\mathbf{p}^{*}\right), x_{2}\left(\mathbf{p}^{*}\right), x_{3}\left(\mathbf{p}^{*}\right)\right)$ for which $\overline{\mathbf{h}}(\mathbf{x})=\mathbf{p}^{*}$ (see Lemma 2.2) (recall the notations $\mathbf{x}^{R}$ and $\mathbf{x}^{E}$, see the second paragraph after Lemma $2.2)$.

We also investigate the replicator dynamics with respect to the population of $\mathbf{e}_{1}, \mathbf{e}_{2}, \mathbf{e}_{3}$ and $\mathbf{p}^{*}=(1 / 3,1 / 3,1 / 3)$ individuals. This means the extension of the dynamics (2.6) adding a forth equation:

$$
\begin{aligned}
& \dot{x}_{1}=x_{1}\left[W_{1}(\mathbf{x})-\bar{W}(\mathbf{x})\right] \\
& \dot{x}_{2}=x_{2}\left[W_{2}(\mathbf{x})-\bar{W}(\mathbf{x})\right] \\
& \dot{x}_{3}=x_{3}\left[W_{3}(\mathbf{x})-\bar{W}(\mathbf{x})\right] \\
& \dot{x}_{4}=x_{4}\left[W_{4}(\mathbf{x})-\bar{W}(\mathbf{x})\right]
\end{aligned}
$$

where $x_{4}$ describes the frequency of $\mathbf{p}^{*}$ individuals. Since $x_{1}+x_{2}+x_{3}+x_{4}=1$, one of equations of the differential equation system can again be omitted. The phase space of this dynamics is $S_{4}$. The state $(1,0,0,0)$ corresponds to pure strategy $\mathbf{e}_{1}$ in the sense that in this state every individual follows strategy $\mathbf{e}_{1}$. Similarly, the state $(0,1,0,0)$ corresponds to pure strategy $\mathbf{e}_{2}$, the state $(0,0,1,0)$ corresponds to pure strategy $\mathbf{e}_{3}$ and the state $(0,0,0,1)$ corresponds to strategy $\mathbf{p}^{*}$. Clearly, if the replicator dynamics (3.10) is restricted to the face with vertices $(1,0,0,0),(0,1,0,0)$ and $(0,0,1,0)$ we get back the dynamics (2.6). Accordingly, this face contains the state $\mathbf{x}^{E}(1 / 3,1 / 3,1 / 3,0)$ ( $\mathbf{x}_{\mathbf{p}^{*}}$ for short) corresponding to the UESS $\mathbf{p}^{*}$ in which each individual follows one of the three pure strategies and the average strategy of the active individuals is the UESS $\mathbf{p}^{*}$. Note that this state is the same as the state $\mathbf{x}^{R}\left(\mathbf{p}^{*}\right)$ for the replicator dynamics with respect to the pure strategies $\mathbf{e}_{1}, \mathbf{e}_{2}, \mathbf{e}_{3}$.

By Lemma 2.2, the segment (the green segment in Figure 2, 4 and 6) connecting $\mathbf{x}_{\mathbf{p}^{*}}$ with $(0,0,0,1)$ precisely consists of the states corresponding to the UESS $\mathbf{p}^{*}$ and the coordinates of these states are $x_{i}^{E}(t / 3, t / 3, t / 3,(1-t))=(t / 3) \rho\left(\mathbf{p}^{*}\right) / \rho_{\mathbf{e}_{i}}\left(\mathbf{p}^{*}\right)(i=1,2,3)$ and $x_{4}\left(\mathbf{p}^{*}\right)=$ $(1-t) \rho\left(\mathbf{p}^{*}\right) / \rho_{\mathbf{p}^{*}}\left(\mathbf{p}^{*}\right)=1-t$, respectively, where $t$ runs through the interval $[0,1]$. These states are equilibrium points of the replicator dynamics (3.10) (Lemma 3.2 in Garay et al. 2018). Furthermore, the state $(0,0,0,1)$ in which every individual of the population follows strategy $\mathbf{p}^{*}$ is a stable (but not asymptotically stable) rest point of the replicator dynamics (3.10) (Theorem 4.9 in Varga et al. 2020). Moreover, if the dynamics (3.10) is restricted to any of the faces with vertex $(0,0,0,1)$ then the state $(0,0,0,1)$ is asymptotically stable on that face (Theorem 4.7 in Varga et al. 2020).

One of the eigenvalues at every point of the segment is always 0 , the other two of them has zero real part in a single point of the segment which is denoted by $\mathbf{x}_{0}$ (the upper green point in Figure 2, 4 and 6). Above the point (the part of the segment falling between the states $\mathbf{x}_{0}$ and $\left.(0,0,0,1)\right)$ both non-zero eigenvalues have negative real parts while under the point (the part of the segment falling between the states $\mathbf{x}_{\mathbf{p}^{*}}$ and $\mathbf{x}_{0}$ ) the real parts of the two non-zero eigenvalues are positive. To find $\mathbf{x}_{0}$ we have analyzed the linearization of the right hand side of the dynamical system (3.10) along the segment between $\mathbf{x}_{\mathbf{p}^{*}}$ and $(0,0,0,1)$. As mentioned, the segment consists of states of form $t \mathbf{x}_{\mathbf{p}^{*}}+(1-t)(0,0,0,1)$ where $t$ runs over the interval $[0,1]$. Recall that the $i$-th coordinate of $\mathbf{x}^{E}$ is calculated according to $(2.4)$, that is,

$$
\mathbf{x}^{E}(t / 3, t / 3, t / 3,1-t)=t \mathbf{x}_{\mathbf{p}^{*}}+(1-t)(0,0,0,1)=\left(x_{1}^{E}, x_{2}^{E}, x_{3}^{E}, x_{4}^{E}\right)
$$


where

$$
x_{i}^{E}= \begin{cases}\frac{\rho\left(\mathbf{p}^{*}\right)}{\rho_{\mathbf{e}_{i}}\left(\mathbf{p}^{*}\right)} \cdot \frac{t}{3} & \text { if } i=1,2,3 ; \\ \frac{\rho\left(\mathbf{p}^{*}\right)}{\rho_{\mathbf{p}^{*}}\left(\mathbf{p}^{*}\right)} \cdot(1-t)=1-t & \text { if } i=4 .\end{cases}
$$

One can calculate the linearization similar to that was done in (2.9) for the replicator dynamics with respect to $\mathbf{e}_{1}, \mathbf{e}_{2}, \mathbf{e}_{3}$. Note that, however, one should use the maps

$$
\boldsymbol{\theta} \mapsto \mathbf{x}^{E}(\boldsymbol{\theta}) \quad \text { and } \quad \mathbf{x} \mapsto(\overbrace{x_{1} \frac{\varrho_{1}(\mathbf{x})}{\bar{\varrho}(\mathbf{x})}}^{\theta_{1}(\boldsymbol{x})}, \overbrace{x_{2} \frac{\varrho_{2}(\mathbf{x})}{\bar{\varrho}(\mathbf{x})}}^{\theta_{2}(\boldsymbol{x})}, \overbrace{x_{3} \frac{\varrho_{3}(\mathbf{x})}{\bar{\varrho}(\mathbf{x})}}^{\theta_{3}(\boldsymbol{x})} \overbrace{x_{4} \frac{\varrho_{4}(\mathbf{x})}{\bar{\varrho}(\mathbf{x})}}^{\theta_{4}(\boldsymbol{x})})=: \boldsymbol{\theta}(\boldsymbol{x}),
$$

respectively, in this case rather than the maps $\mathbf{p} \mapsto \mathbf{x}^{R}(\mathbf{p})$ and $\mathbf{x} \mapsto \overline{\mathbf{h}}(\mathbf{x})$. Accordingly, we rewrite the dynamics $(3.10) \dot{x}_{i}=x_{i}\left[W_{i}(\mathbf{x})-\bar{W}(\mathbf{x})\right](i=1,2,3)$ as

$$
\dot{x}_{i}=\theta_{i}(\mathbf{x}) \frac{\rho(\overline{\mathbf{h}}(\mathbf{x}))}{\rho_{\mathbf{e}_{i}}(\overline{\mathbf{h}}(\mathbf{x}))}\left[\omega_{\mathbf{e}_{i}}(\overline{\mathbf{h}}(\mathbf{x}))-\omega(\overline{\mathbf{h}}(\mathbf{x}))\right] \quad(i=1,2,3) .
$$

[Note that $\overline{\mathbf{h}}(\mathbf{x})=\theta_{1}(\mathbf{x}) \mathbf{e}_{1}+\theta_{2}(\mathbf{x}) \mathbf{e}_{2}+\theta_{3}(\mathbf{x}) \mathbf{e}_{3}+\theta_{4}(\mathbf{x}) \mathbf{p}^{*}$ by (2.3) and (3.11).] Also, the functions $R_{i}(i=1,2,3)$ should be given as the functions of the coefficient-list $\boldsymbol{\theta}=\left(\theta_{1}, \theta_{2}, \theta_{3}, \theta_{4}\right)$, that is,

$$
R_{i}\left(\theta_{1}, \theta_{2}, \theta_{3}\right)=\theta_{i} \frac{\rho(\mathbf{p}(\boldsymbol{\theta}))}{\rho_{\mathbf{e}_{i}}(\mathbf{p}(\boldsymbol{\theta}))}\left[\omega_{\mathbf{e}_{i}}(\mathbf{p}(\boldsymbol{\theta}))-\omega(\mathbf{p}(\boldsymbol{\theta}))\right] \quad(i=1,2,3)
$$

where $\mathbf{p}(\boldsymbol{\theta})=\mathbf{p}\left(\theta_{1}, \theta_{2}, \theta_{3}\right)=\theta_{1} \mathbf{e}_{1}+\theta_{2} \mathbf{e}_{2}+\theta_{3} \mathbf{e}_{3}+\left(1-\theta_{1}-\theta_{2}-\theta_{3}\right) \mathbf{p}^{*}\left(\right.$ since $\theta_{1}+\theta_{2}+\theta_{3}+\theta_{4}=1$, the variable $\theta_{4}$ can be dropped). Then, similar to (2.9), we can calculate the Jacobian matrix along the segment $t \mathbf{x}_{\mathbf{p}^{*}}+(1-t)(0,0,0,1), t \in[0,1]$. After having the linearization along this segment we compute the related characteristic polynomials $\lambda^{3}+b(t) \lambda^{2}+c(t) \lambda+d(t)$. Since one of the eigenvalues is zero at every point of the the segment ${ }^{3}$, it follows that $d(t)=0$ for any $t \in[0,1]$. Therefore the other two eigenvalues should be the zeros of the polynomial $\lambda^{2}+b(t) \lambda+c(t)$. The formulas for $b(t)$ and $c(t)$, respectively, is not too "beautiful" (we, too, have used Wolfram Mathematica 12), but, after analyzing them, one can see that $b(0)=$ $c(0)=0$ and $c(t)>0$ for $t \in(0,1]$. Hence, the real part of the zeros can be zero only if $b(t)=0 . \quad b(t)$ has the form $t\left(b_{11}-b_{12} t\right) /\left(b_{22} t^{2}-b_{21} t+b_{20}\right)$ where the coefficients $b_{i j}$-s are positive, $b_{12}>b_{11}$ and $b_{20}>b_{21}$. We infer that the denominator is positive for any $t \in[0,1]$ so, if $t \in(0,1]$, the sign of $b(t)$ agrees with the sign of the expression $b_{11}-b_{12} t$ which is positive if $0<t<b_{11} / b_{12}$, negative if $b_{11} / b_{12}<t \leq 1$ and 0 if $t=b_{11} / b_{12}$. It follows (recall condition $[\mathrm{C} 4]$ too) that the real parts of the two non-zero eigenvalues are positive if $0<t<b_{11} / b_{12}, 0$ if $t=b_{11} / b_{12}$ and negative if $b_{11} / b_{12}<t \leq 1$. The state belonging to $t=b_{11} / b_{12}$ is $\mathbf{x}_{0}$. The states belonging to $t$-s between 0 and $b_{11} / b_{12}$ forms the segment between $\mathbf{x}_{\mathbf{p}^{*}}$ and $\mathbf{x}_{0}$ while the states belonging to $t$-s between $b_{11} / b_{12}$ and 1 forms the segment between $\mathbf{x}_{0}$ and $(0,0,0,1)$.

We remark that the local property of equilibrium points and the global behaviour of the dynamics on the edges of the phase spaces $S_{3}$ and $S_{4}$, respectively, are exact, the eigenvalues were calculated, whereas the global behaviour in the interior of the phase spaces and in the interior of the faces of $S_{4}$ are just based on numerical simulations of the phase portrait.

\footnotetext{
${ }^{3}$ This is because every point of the segment is a rest point of the dynamics (3.10).
} 


\subsection{Example 1}

The payoff matrix $A$ and the time constraint matrix $T$ are

$$
A=\left(\begin{array}{ccc}
-1 / 2 & 5 & -1 \\
-2 & 5 / 2 & 3 \\
(-17+7 \sqrt{17}) / 8 & 1 & 1 / 4
\end{array}\right), \quad T=\left(\begin{array}{ccc}
0 & 1 & 0 \\
0 & 0 & 1 \\
0 & 0 & 0
\end{array}\right)
$$

The function $f(\mathbf{q})$ defined in (2.5) is a large expression in our examples. To check conditions [C1], [C2] and [C3], therefore, we have used Mathematica 12 and we only give rounded values in the paper. By straightforward calculation, one can check condition [C1]. For the second order derivatives we obtain:

$$
f_{q_{1}, q_{1}}^{\prime \prime}(1 / 3,1 / 3) \approx 0.9024 \quad f_{q_{2}, q_{2}}^{\prime \prime}(1 / 3,1 / 3) \approx 1.569 \quad f_{q_{1}, q_{2}}^{\prime \prime}(1 / 3,1 / 3) \approx 1.0397
$$

from which we get conditions [C2] and [C3]:

$$
f_{q_{1}, q_{1}}^{\prime \prime}(1 / 3,1 / 3) f_{q_{2}, q_{2}}^{\prime \prime}(1 / 3,1 / 3) \approx 1.416>1.081 \approx\left[f_{q_{1}, q_{2}}^{\prime \prime}(1 / 3,1 / 3)\right]^{2} .
$$

The phase portrait of the dynamics (2.8) with respect to $\mathbf{e}_{1}, \mathbf{e}_{2}, \mathbf{e}_{3}$ is depicted in Figure 1. The three vertices together with the edges between them form a heteroclinic cycle. The equilibrium point $\mathbf{x}\left(\mathbf{p}^{*}\right)=\mathbf{x}^{R}\left(\mathbf{p}^{*}\right)=((7-\sqrt{17}) / 8,(7-\sqrt{17}) / 8,(2 \sqrt{17}-6) / 8)$ corresponding to the UESS $\mathbf{p}^{*}=(1 / 3,1 / 3,1 / 3)$ has eigenvalues with positive real part. Condition [C4] is true:

$$
b=\frac{-1555+377 \sqrt{17}}{16} \approx-0.0368<0 \quad \text { and } \quad c=-\frac{567}{2}+\frac{551 \sqrt{17}}{8} \approx 0.4789 .
$$

The phase portrait suggest that the solutions tend to the boundary of the simplex.

The phase portrait of the extended replicator dynamics (3.10) is shown in Figure 2. On the face determined by the vertices $(1,0,0,0),(0,1,0,0),(0,0,1,0)$, the dynamics agrees with (2.7) so the phase portrait on it is the same as in Figure 1.

There is an equilibrium point on the edge between the vertices $(0,1,0,0)$ and $(0,0,0,1)$ with coordinates $x_{2}^{(24)} \approx 0.3942, x_{1}^{(24)}=x_{3}^{(24)}=0, x_{4}^{(24)}=1-x_{2}^{(24)}$. It is denoted by $\mathbf{x}^{(24)}$ This equilibrium is unstable with respect to the dynamics restricted to the edge.

The solutions in the interior of the face determined by the vertices $(1,0,0,0),(0,1,0,0)$, $(0,0,0,1)$ start from the equilibrium point $\mathbf{x}^{(24)}$ and end in $(0,0,0,1)$.

The solutions in the interior of the face determined by the vertices $(1,0,0,0),(0,0,1,0)$, $(0,0,0,1)$ start from $(1,0,0,0)$ and end in $(0,0,0,1)$.

On the face determined by the vertices $(0,1,0,0),(0,0,1,0),(0,0,0,1)$, there is a separatrix starting from $(0,0,1,0)$ and ending in the equilibrium point $\mathbf{x}^{(24)}$. The solutions falling toward the edge between the $(0,1,0,0)$ and $(0,0,1,0)$ from the separatrix start from $(0,0,1,0)$ and end in $(0,1,0,0)$ while those on the $(0,0,0,1)$ side of the separatrix start from $(0,0,1,0)$ and end in $(0,0,0,1)$.

The state $\mathbf{x}_{0}$ on the segment matching $\mathbf{x}_{\mathbf{p}^{*}}$ with $(0,0,0,1)$ and corresponding to the UESS $\mathbf{p}^{*}$ is equal to $\mathbf{x}^{E}\left(t_{0} / 3, t_{0} / 3, t_{0} / 3,1-t_{0}\right)$ where $t_{0}=(175+544 \sqrt{17}) / 2957 \approx 0.8177$. Accordingly, the coordinates of $\mathbf{x}_{0}$ are $\left(x_{0}\right)_{1} \approx 0.2941,\left(x_{0}\right)_{2} \approx 0.2941,\left(x_{0}\right)_{3} \approx 0.2296$ and $\left(x_{0}\right)_{4}=1-t_{0} \approx 0.1823$. 


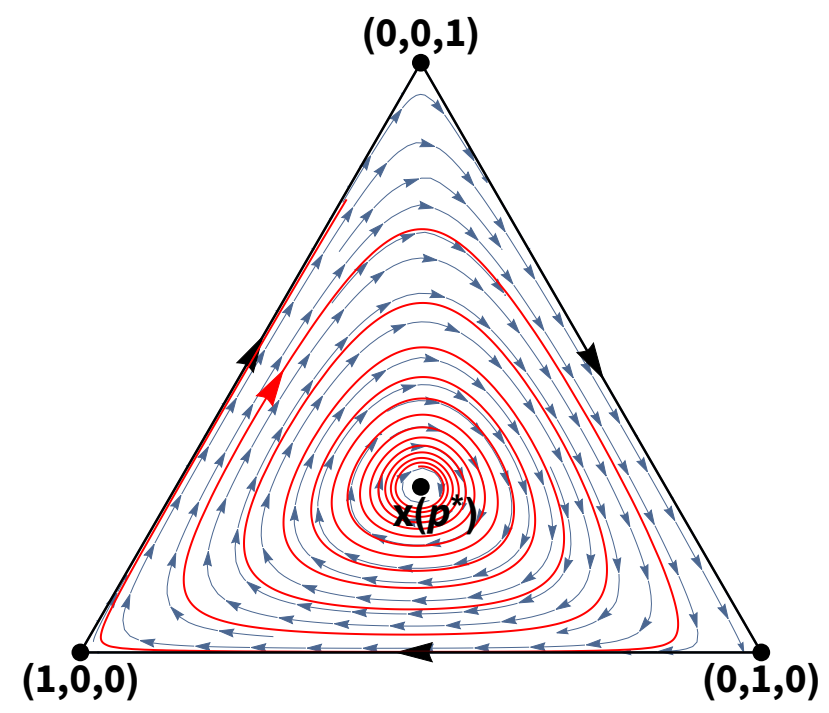

\begin{tabular}{|c|c|c|}
\hline State & \multicolumn{2}{|c|}{ Real part of the eigenvalues } \\
\hline$(1,0,0)$ & -1.5 & 1.983 \\
\hline$(0,1,0)$ & -1.5 & 0 \\
\hline$(0,0,1)$ & -1.25 & 1.25 \\
\hline $\mathbf{x}\left(\mathbf{p}^{*}\right)$ & 0.01841 & 0.01841 \\
\hline
\end{tabular}

Figure 1. The phase portrait of the replicator dynamics (2.6) with respect to the pure phenotypes $\mathbf{e}_{1}, \mathbf{e}_{2}$ and $\mathbf{e}_{3}$. The payoff matrix A and the time constraint matrix $\mathrm{T}$ are the matrices in (3.12). $\mathbf{x}\left(\mathbf{p}^{*}\right)=\mathbf{x}^{R}\left(\mathbf{p}^{*}\right)$ is the state corresponding to strategy $\mathbf{p}^{*}=(1 / 3,1 / 3,1 / 3)$ through Lemma $2.2: x_{1}\left(\mathbf{p}^{*}\right)=x_{2}\left(\mathbf{p}^{*}\right)=(7-\sqrt{17}) / 8 \approx 0.36$. Although $\mathbf{p}^{*}$ is a UESS with the matrix games under time constraints, $\mathbf{x}\left(\mathbf{p}^{*}\right)$ is an unstable rest point of the replicator dynamics. The vertices of the simplex are saddle points and the boundary of the simplex is a heteroclinic cycle which appears to be a stable limit cycle. In the table, we give the real parts of the eigenvalues of the linearization of the replicator dynamics at the rest points indicated in the phase portrait.

\subsection{Example 2}

The payoff matrix $A$ and the time constraint matrix $T$ are

$$
A=\left(\begin{array}{ccc}
-3 / 5 & (2921-19 \sqrt{97}) / 270 & -1 \\
-2 & 66 / 10 & 3 \\
(-86+19 \sqrt{97}) / 54 & 1 & 1 / 10
\end{array}\right), \quad T=\left(\begin{array}{lll}
4 & 4 & 4 \\
4 & 2 & 4 \\
0 & 0 & 0
\end{array}\right) .
$$

If we calculate the first order derivatives of function $f$ in (2.5) we can see that condition [C1] hold. For the second order derivatives, we get

$$
f_{q_{1}, q_{1}}^{\prime \prime}(1 / 3,1 / 3) \approx 0.05508 \quad f_{q_{2}, q_{2}}^{\prime \prime}(1 / 3,1 / 3) \approx 0.1041 \quad f_{q_{1}, q_{2}}^{\prime \prime}(1 / 3,1 / 3) \approx-0.02954
$$

from which we gain that conditions $[\mathrm{C} 2]$ and $[\mathrm{C} 3]$ also hold:

$$
f_{q_{1}, q_{1}}^{\prime \prime}(1 / 3,1 / 3) f_{q_{2}, q_{2}}^{\prime \prime}(1 / 3,1 / 3) \approx 0.005732>8.726 \cdot 10^{-4} \approx\left[f_{q_{1}, q_{2}}^{\prime \prime}(1 / 3,1 / 3)\right]^{2} .
$$

At first sight, the phase portraits are similar to those of the previous example. However, it appears an asymptotically stable periodic orbit on the phase portrait of the replicator dynamics with respect to the pure strategies while the boundary of the simplex is now an unstable heteroclinic cycle (see Figure 3 ). The unstable rest point $\mathbf{x}\left(\mathbf{p}^{*}\right)$ corresponding to the UESS $\mathbf{p}^{*}=(1 / 3,1 / 3,1 / 3)$ has the coordinates $x_{1}\left(\mathbf{p}^{*}\right)=(285-7 \sqrt{97}) / 484 \approx 0.4464$, $x_{2}\left(\mathbf{p}^{*}\right)=(58-\sqrt{97}) / 121 \approx 0.3979, x_{3}\left(\mathbf{p}^{*}\right)=1-x_{1}\left(\mathbf{p}^{*}\right)-x_{2}\left(\mathbf{p}^{*}\right)$. Condition [C4] holds with

$$
\begin{aligned}
& b=\frac{-38727752+3910015 \sqrt{97}}{99305910} \approx-0.002201 \quad \text { and } \\
& c=\frac{7(59852935537-5238596975 \sqrt{97})}{643502296800} \approx 0.08984 .
\end{aligned}
$$


bioRxiv preprint doi: https://doi.org/10.1101/2021.08.05.455237; this version posted August 6, 2021. The copyright holder for this preprint (which was not certified by peer review) is the author/funder, who has granted bioRxiv a license to display the preprint in perpetuity. It is made available under aCC-BY-NC-ND 4.0 International license.

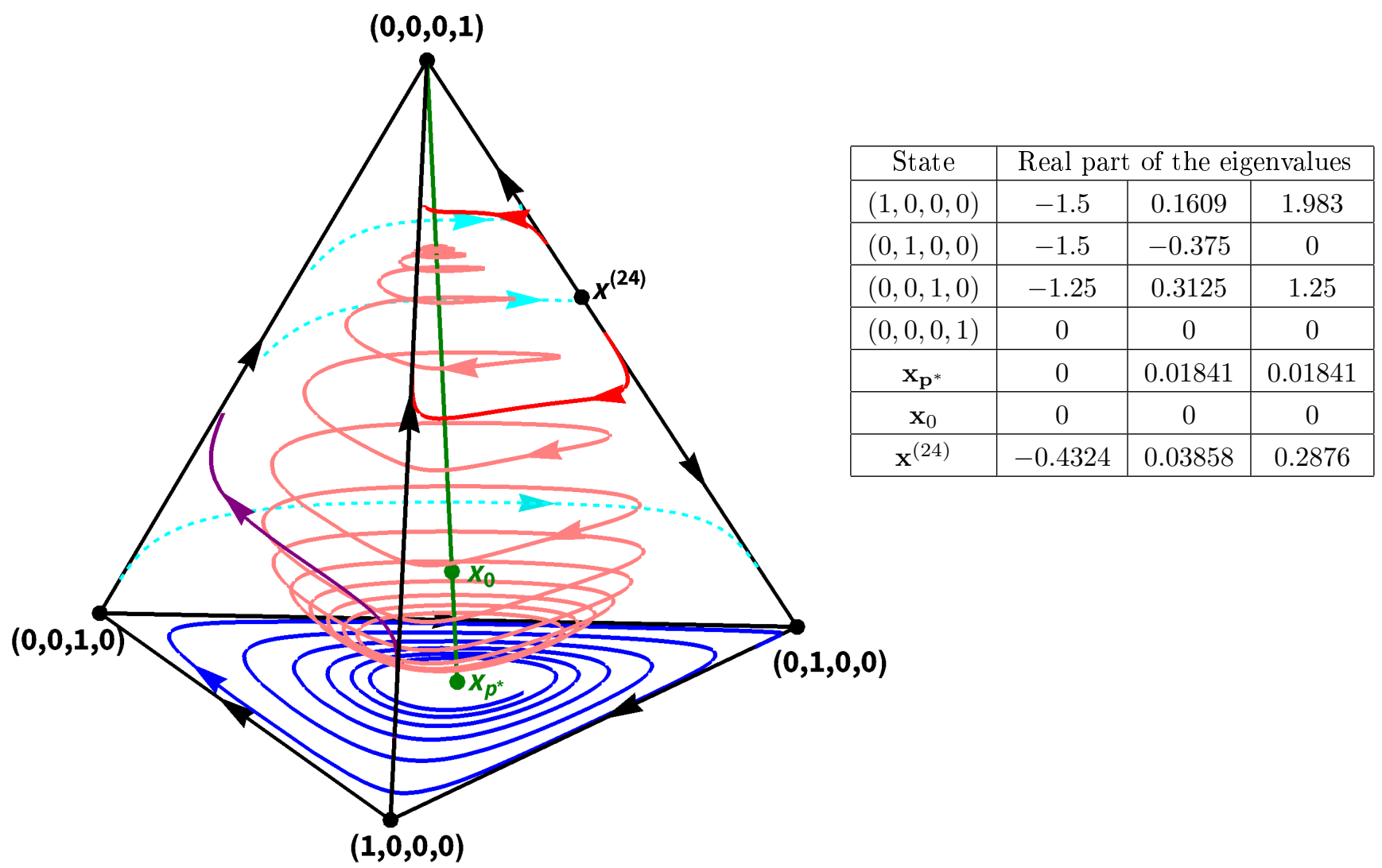

Figure 2. The phase portrait of the replicator dynamics with respect to phenotypes $\mathbf{e}_{1}, \mathbf{e}_{2}, \mathbf{e}_{3}$ and $\mathbf{p}^{*}=(1 / 3,1 / 3,1 / 3)$. The payoff matrix $\mathrm{A}$ and the time constraint matrix $\mathrm{T}$ are given in $(3.12) . \mathbf{x}_{\mathbf{p}^{*}}=\left(x_{\mathbf{p}^{*}, 1}, x_{\mathbf{p}^{*}, 2}, x_{\mathbf{p}^{*} 3}, x_{\mathbf{p}^{*}, 4}\right)$ is the state corresponding to strategy $\mathbf{p}^{*}=(1 / 3,1 / 3,1 / 3)$ through Lemma 2.2 on the face determined by the vertices $(1,0,0,0)$, $(0,1,0,0)$ and $(0,0,1,0): x_{\mathbf{p}^{*}, 1}=x_{\mathbf{p}^{*}, 2}=(7-\sqrt{17}) / 8 \approx 0.36, x_{\mathbf{p}^{*}, 3}=1-x_{\mathbf{p}^{*}, 1}-x_{\mathbf{p}^{*}, 2}, x_{\mathbf{p}^{*}, 4}=0$. It agrees with $\mathbf{x}\left(\mathbf{p}^{*}\right)$ in Figure 1. Every state on the green segment between $(0,0,0,1)$ and $\mathbf{x}_{\mathbf{p}^{*}}$ corresponds to strategy $\mathbf{p}^{*}$ through Lemma 2.2. Hence every point of the segment is a rest point of the replicator dynamics. One of the three eigenvalues of the linearization of the replicator dynamics (3.10) at these states is zero. At $\mathbf{x}_{0}$, all of the eigenvalues have zero real part. The states on the segment under $\mathbf{x}_{0}$ have two eigenvalues with positive real part, so they are all unstable though they correspond to the UESS $\mathbf{p}^{*}$. The states between $\mathbf{x}_{0}$ and $(0,0,0,1)$ whereas have two eigenvalues with negative real part. The state $(0,0,0,1)$ is stable (but not asymptotically). In the table, we give the real parts of the eigenvalues of the linearization of the replicator dynamics at the rest points indicated in the phase portrait.

The phase portrait of the extended dynamics (3.10) is depicted in Figure 4. On the face determined by the vertices $(1,0,0,0),(0,1,0,0)$ and $(0,0,1,0)$, the extended dynamics agrees with the dynamics with respect to the pure strategies. Here the rest point corresponding to the UESS $\mathbf{p}^{*}$ is denoted by $\mathbf{x}_{\mathbf{p}^{*}}=\mathbf{x}^{E}(1 / 3,1 / 3,1 / 3,0)$.

On the other three faces of the simplex the dynamics behaves similar to that in example 1. The coordinates of the rest point denoted by $\mathbf{x}^{(24)}$ on the edge between $(0,1,0,0)$ and $(0,0,0,1)$ are $x_{1}^{(24)}=x_{3}^{(24)}=0, x_{2}^{(24)} \approx 0.1176$ and $x_{4}^{(24)}=1-x_{2}^{(24)}$.

The state $\mathbf{x}_{0}$ is now the state $\mathbf{x}^{E}\left(t_{0} / 3, t_{0} / 3, t_{0} / 3,1-t_{0}\right)$ where

$$
t_{0}=\frac{17(-391107+83251 \sqrt{97}}{8819456} \approx 0.8266
$$

and so the coordinates of $\mathbf{x}_{0}$ are $\left(x_{0}\right)_{1} \approx 0.369,\left(x_{0}\right)_{2} \approx 0.3289,\left(x_{0}\right)_{3} \approx 0.1287$ and $\left(x_{0}\right)_{4}=$ $1-t_{0} \approx 0.1734$. 


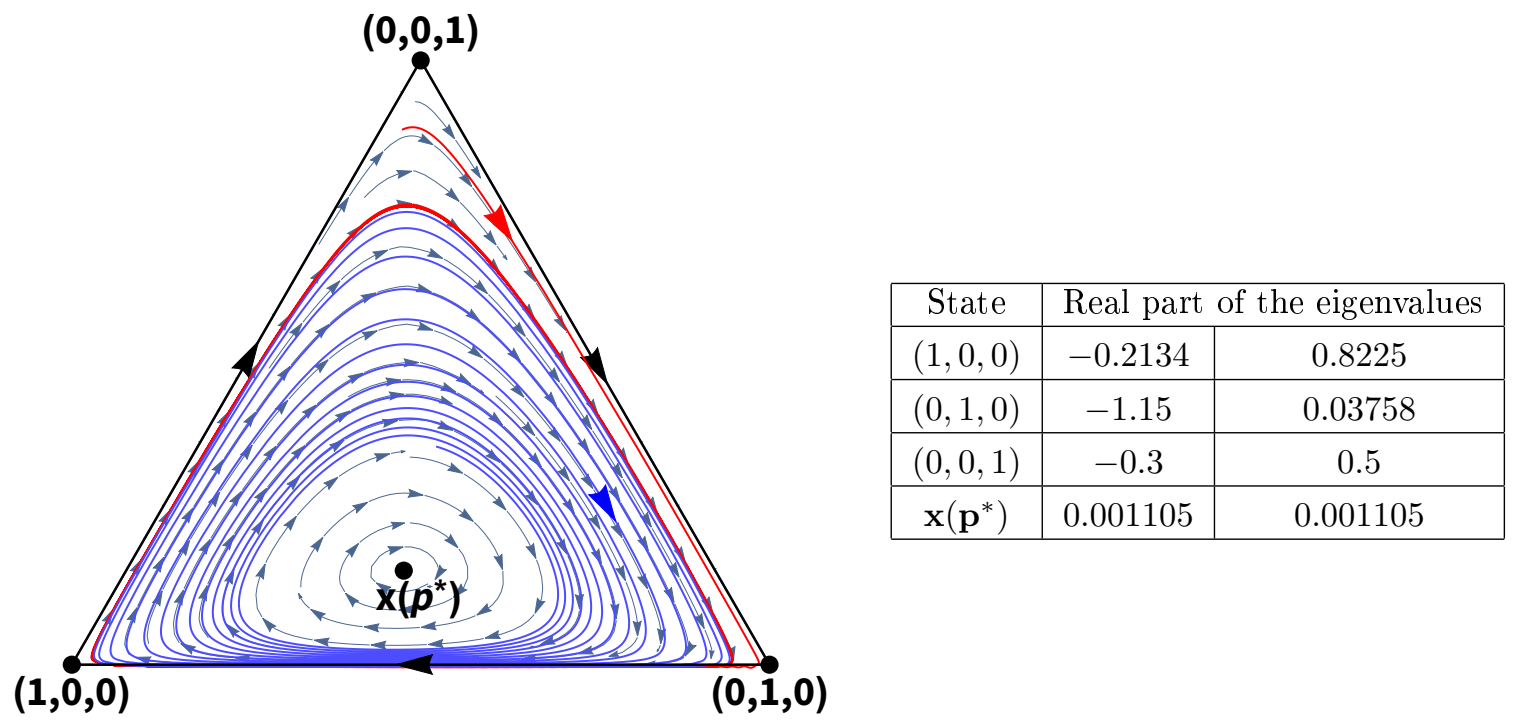

Figure 3. The phase portrait of the replicator dynamics (2.6) with respect to the pure phenotypes $\mathbf{e}_{1}, \mathbf{e}_{2}$ and $\mathbf{e}_{3}$. The payoff matrix $\mathrm{A}$ and the time constraint matrix $\mathrm{T}$ are the matrices in $(3.13) . \mathbf{x}\left(\mathbf{p}^{*}\right)=\mathbf{x}^{R}\left(\mathbf{p}^{*}\right)$ is the state corresponding to strategy $\mathbf{p}^{*}=(1 / 3,1 / 3,1 / 3)$ through Lemma $2.2: x_{1}\left(\mathbf{p}^{*}\right)=(285-7 \sqrt{97}) / 484 \approx 0.446, x_{2}\left(\mathbf{p}^{*}\right)=(58-\sqrt{97}) / 121 \approx 0.398$. Although $\mathbf{p}^{*}$ is a UESS with the matrix games under time constraints, $\mathbf{x}\left(\mathbf{p}^{*}\right)$ is an unstable rest point of the replicator dynamics. The vertices of the simplex are saddle points and the boundary of the simplex is a heteroclinic cycle similar to example 1 but it appears to be an unstable limit cycle, whereas it seems that there is a stable periodic orbit near to the boundary of the simplex. In the table, we give the real parts of the eigenvalues of the linearization of the replicator dynamics at the rest points indicated in the phase portrait.

\subsection{Example 3}

The payoff matrix $A$ and the time constraint matrix $T$ are the following in this case:

$$
A=\left(\begin{array}{ccc}
-5 & 449-19 \sqrt{469} & -1 \\
-2 & 5 & 3 \\
-204 / 5+2 \sqrt{469} & 1 & -2 / 10
\end{array}\right), \quad T=\left(\begin{array}{ccc}
45 & 40 & 20 \\
1 & 2 & 7 \\
0 & 0 & 0
\end{array}\right) .
$$

Calculating the first order derivatives of function $f$ in $(2.5)$ at $\mathbf{q}=(1 / 3,1 / 3)$ we get that both of them are zero so condition [C1] is valid. The second order derivatives are

$$
f_{q_{1}, q_{1}}^{\prime \prime}(1 / 3,1 / 3) \approx 1.154 \quad f_{q_{2}, q_{2}}^{\prime \prime}(1 / 3,1 / 3) \approx 0.1341 \quad f_{q_{1}, q_{2}}^{\prime \prime}(1 / 3,1 / 3) \approx-0.3665 .
$$

Hence we infer that conditions [C2] and [C3] are also satisfied:

$$
f_{q_{1}, q_{1}}^{\prime \prime}(1 / 3,1 / 3) f_{q_{2}, q_{2}}^{\prime \prime}(1 / 3,1 / 3) \approx 0.1547>0.1343 \approx\left[f_{q_{1}, q_{2}}^{\prime \prime}(1 / 3,1 / 3)\right]^{2} .
$$

The most complex phase portrait belongs to this case. In addition to $\mathbf{p}^{*}=(1 / 3,1 / 3,1 / 3)$, there is a further UESS, moreover, a strict Nash equilibrium. A strategy $\mathbf{r}$ is a strict Nash equilibrium if, for every strategy $\mathbf{q} \neq \mathbf{r}$, the strict inequality

$$
\rho_{\mathbf{q}}(\mathbf{r}) \mathbf{q} A \mathbf{r}<\rho(\mathbf{r}) \mathbf{r} A \mathbf{r}
$$

holds (Definition 2.2 in Garay et al. 2018). If $\mathbf{r}$ is a strict Nash equilibrium then it is a pure strategy (Theorem 4.1 in Garay et al. 2018) and a UESS (Theorem 4.1 in Varga et al. 2020). For this case, strategy $\mathbf{e}_{2}$ is a strict Nash equilibrium implying that the corresponding state $(0,1,0)$ and $(0,1,0,0)$, respectively, is an asymptotically stable rest point of the replicator dynamics with respect to $\mathbf{e}_{1}, \mathbf{e}_{2}, \mathbf{e}_{3}$ and the replicator dynamics with respect to $\mathbf{e}_{1}, \mathbf{e}_{2}, \mathbf{e}_{3}, \mathbf{p}^{*}$, respectively (Corollary 4.8 in Varga et al. 2020). 
bioRxiv preprint doi: https://doi.org/10.1101/2021.08.05.455237; this version posted August 6, 2021. The copyright holder for this preprint (which was not certified by peer review) is the author/funder, who has granted bioRxiv a license to display the preprint in perpetuity. It is made available under aCC-BY-NC-ND 4.0 International license.

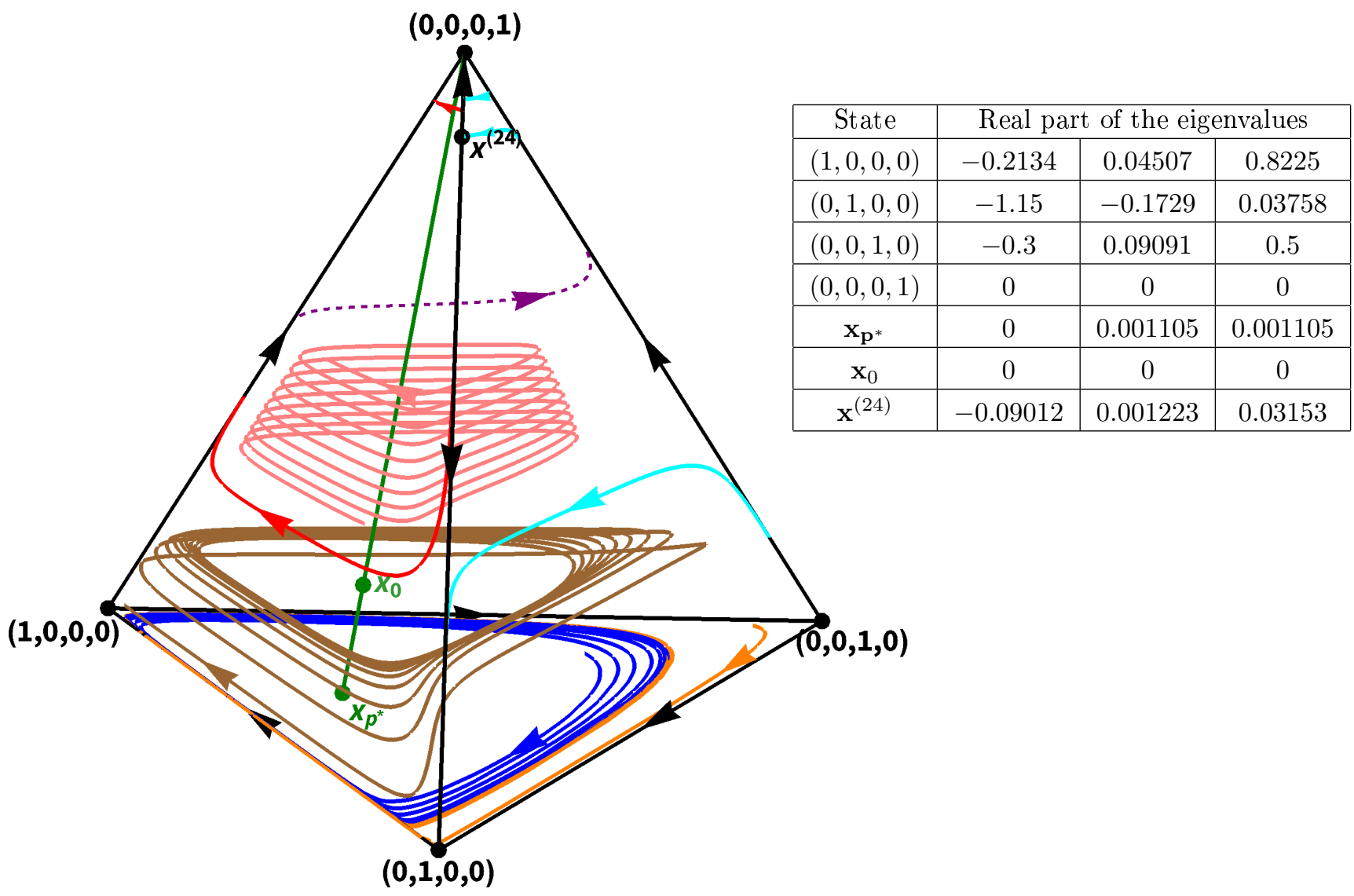

Figure 4. The phase portrait of the replicator dynamics with respect to phenotypes $\mathbf{e}_{1}, \mathbf{e}_{2}, \mathbf{e}_{3}$ and $\mathbf{p}^{*}=(1 / 3,1 / 3,1 / 3)$. The payoff matrix $\mathrm{A}$ and the time constraint matrix $\mathrm{T}$ are given in $(3.13) . \mathbf{x}_{\mathbf{p}^{*}}=\left(x_{\mathbf{p}^{*}, 1}, x_{\mathbf{p}^{*}, 2}, x_{\mathbf{p}^{*}, 3}, x_{\mathbf{p}^{*}, 4}\right)$ is the state corresponding to strategy $\mathbf{p}^{*}=(1 / 3,1 / 3,1 / 3)$ through Lemma 2.2 on the face determined by the vertices $(1,0,0,0)$, $(0,1,0,0)$ and $(0,0,1,0): x_{\mathbf{p}^{*}, 1} \approx 0.446, x_{\mathbf{p}^{*}, 2} \approx 0.398, x_{\mathbf{p}^{*}, 3}=1-x_{\mathbf{p}^{*}, 1}-x_{\mathbf{p}^{*}, 2}, x_{\mathbf{p}^{*} 4}=0$. It agrees with $\mathbf{x}\left(\mathbf{p}^{*}\right)$ in Figure 3 . Every state on the green segment between $(0,0,0,1)$ and $\mathbf{x}_{\mathbf{p}} *$ corresponds to strategy $\mathbf{p}^{*}$ through Lemma 2.2 . Hence every point of the segment is a rest point of the replicator dynamics. One of the three eigenvalues of the linearization of the replicator dynamics (3.10) at these states is zero. At $\mathbf{x}_{0}$, all of the eigenvalues have zero real part. The other two eigenvalues of the states on the segment under $\mathbf{x}_{0}$ have positive real part, so they are all unstable though they correspond to the UESS $\mathbf{p}^{*}$. The states between $\mathbf{x}_{0}$ and $(0,0,0,1)$ whereas have two eigenvalues with negative real part. The state $(0,0,0,1)$ is stable (but not asymptotically). In the table, we give the real parts of the eigenvalues of the linearization of the replicator dynamics at the rest points indicated in the phase portrait.

To see that $\mathbf{e}_{2}$ is a strict Nash equilibrium one should check inequality (3.15) with $\mathbf{r}=\mathbf{e}_{2}$. By (2.1) and (2.2) we get $\rho\left(\mathbf{e}_{2}\right)$ and $\rho_{\mathbf{q}}\left(\mathbf{e}_{2}\right)$, respectively. We obtain that

$$
\rho\left(\mathbf{e}_{2}\right) \mathbf{e}_{2} A \mathbf{e}_{2}-\rho_{\mathbf{q}}\left(\mathbf{e}_{2}\right) \mathbf{q} A \mathbf{e}_{2}=\frac{3-796 q_{1}+38 \sqrt{469} q_{1}-3 q_{2}}{2+40 q_{1}+2 q_{2}}
$$

Note that $\mathbf{q}=\left(q_{1}, q_{2}, 1-q_{1}-q_{2}\right) \in S_{3}$ is a strategy. Thus the denominator $2+40 q_{1}+2 q_{2}$ is positive for every $\mathbf{q}$. Consequently, in order to prove that $\mathbf{e}_{2}$ is a strict Nash equilibrium, it is enough to validate that the numerator $3-796 q_{1}+38 \sqrt{469} q_{1}-3 q_{2}>0$ for every $\mathbf{q} \neq \mathbf{e}_{2}$ which can easily be seen:

$$
\begin{aligned}
3-796 q_{1}+38 \sqrt{469} q_{1}-3 q_{2} \geq 3-796 q_{1}+38 \sqrt{441} q_{1}-3 q_{2} & =3+2 q_{1}-3 q_{2} \\
& =2 q_{1}+3\left(1-q_{2}\right)
\end{aligned}
$$

which is positive for every strategy $\mathbf{q}$ different from $\mathbf{e}_{2}$. Hence $\mathbf{e}_{2}$ is a strict Nash equilibrium, as claimed. 


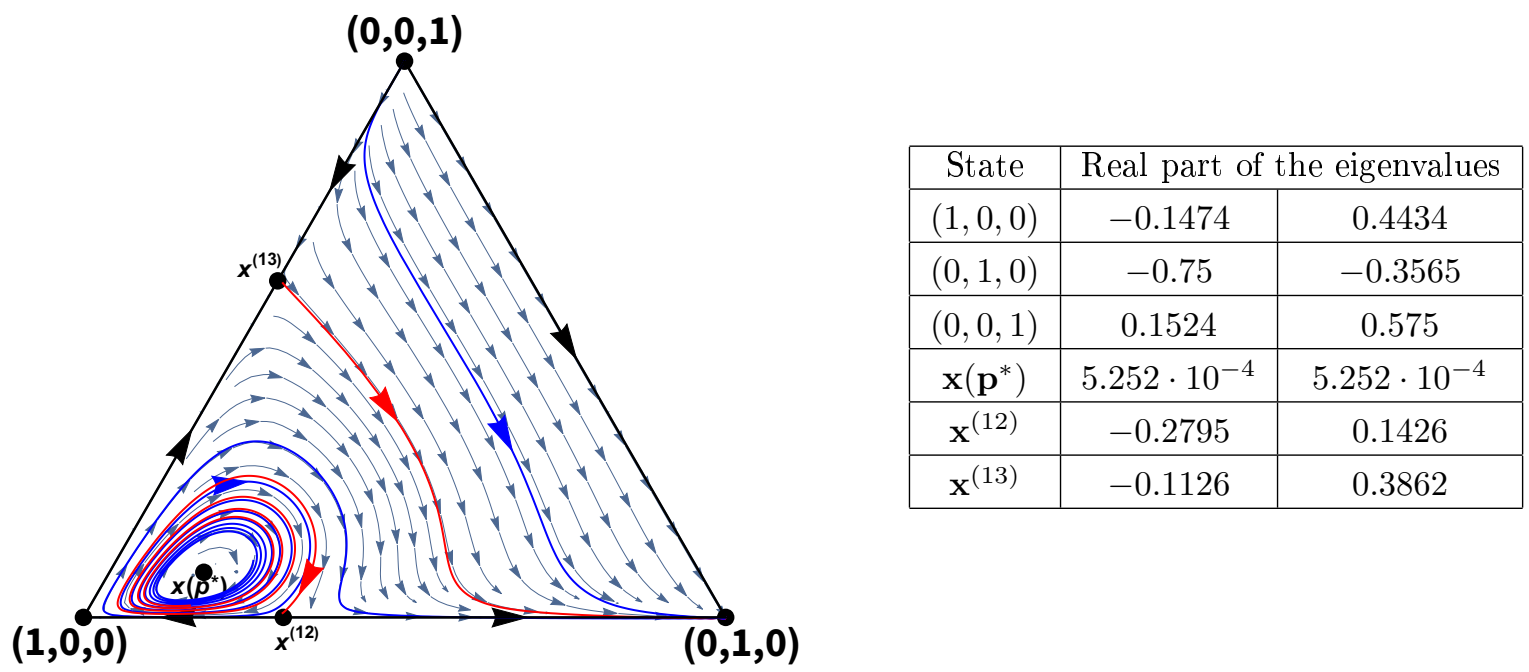

Figure 5. The phase portrait of the replicator dynamics (2.6) with respect to the pure phenotypes $\mathbf{e}_{1}, \mathbf{e}_{2}$ and $\mathbf{e}_{3}$. The payoff matrix $A$ and the time constraint matrix $T$ are the matrices in $(3.14) . \mathbf{x}\left(\mathbf{p}^{*}\right)=\mathbf{x}^{R}\left(\mathbf{p}^{*}\right)$ is the state corresponding to strategy $\mathbf{p}^{*}=(1 / 3,1 / 3,1 / 3)$ through Lemma $2.2: x_{1}\left(\mathbf{p}^{*}\right)=(495-4 \sqrt{469}) / 529 \approx 0.772, x_{2}\left(\mathbf{p}^{*}\right)=(409+17 \sqrt{469}) / 5290 \approx$ 0.147. Although $\mathbf{p}^{*}$ is a UESS with the matrix games under time constraints, $\mathbf{x}\left(\mathbf{p}^{*}\right)$ is an unstable rest point of the replicator dynamics. Among the vertices, only $(1,0,0)$ is a saddle point; $(0,1,0)$ is asymptotically stable, moreover, it corresponds to a strict Nash equilibrium while $(0,0,1)$ is a source. In addition to the vertices, $\mathbf{x}^{(12)}$ and $\mathbf{x}^{(13)}$ are two further rest points on the boundary of the simplex. Both of them are saddle points. In the table, we give the real parts of the eigenvalues of the linearization of the replicator dynamics at the rest points indicated in the phase portrait.

Considering the replicator dynamics with respect to $\mathbf{e}_{1}, \mathbf{e}_{2}$ and $\mathbf{e}_{3}$, we find two further rest points besides the vertices and the rest point $\mathbf{x}^{R}\left(\mathbf{p}^{*}\right)$ corresponding to the UESS $\mathbf{p}^{*}$. There is a rest point on the edge between the states $(1,0,0)$ and $(0,1,0)$. We denote it by $\mathbf{x}^{(12)} \approx(0.689,0.311,0)$. This is an unstable rest point of the dynamics restricted to the edge and it is a saddle point of the dynamics with respect to the pure strategies.

The other rest point can be found on the edge between $(1,0,0)$ and $(0,0,1)$. It is denoted by $\mathbf{x}^{(13)} \approx(0.395,0,0.605)$. This state is asymptotically stable on the edge but it is a saddle point of the replicator dynamics with respect to $\mathbf{e}_{1}, \mathbf{e}_{2}$ and $\mathbf{e}_{3}$. A separatrix starts from it and ends in the state $(0,1,0)$.

The (interior) solutions on the $(1,0,0)$ side of the separatrix start from the state $\mathbf{x}\left(\mathbf{p}^{*}\right)=$ $\mathbf{x}^{R}\left(\mathbf{p}^{*}\right)$ and end in $(0,1,0)$ except for one which ends in $\mathbf{x}^{(12)}$. The (interior) solutions on the $(0,0,1)$ side of the separatrix start from $(0,0,1)$ and end in $(0,1,0)$ (see Figure 5).

The coordinates of $\mathbf{x}\left(\mathbf{p}^{*}\right)$ are $x_{1}\left(\mathbf{p}^{*}\right)=(495-4 \sqrt{469}) / 529 \approx 0.772, x_{2}\left(\mathbf{p}^{*}\right)=(409+$ $17 \sqrt{469}) / 5290 \approx 0.1469$ and condition [C4] holds with

$$
\begin{aligned}
& b=\frac{-4707833609+217368843 \sqrt{469}}{385839375} \approx-0.00105<0 \quad \text { and } \\
& c=\frac{37(-6522761117+301243109 \sqrt{469})}{1929196875} \approx 0.02076 .
\end{aligned}
$$

The phase portrait of the extended replicator dynamics (3.10) is illustrated in Figure 6 . The restriction of the dynamics onto the face determined by the vertices $(1,0,0,0),(0,1,0,0)$ and $(0,0,1,0)$ is just the replicator dynamics with respect to the pure strategies so the phase portrait on that face agrees with that in Figure 5.

As in the first two examples, there exist again a rest point on the edge between $(0,1,0,0)$ and $(0,0,0,1)$. It is denoted by $\mathbf{x}^{(24)}$. Its coordinates are $x_{1}^{(24)}=x_{3}^{(24)}=0, x_{2}^{(24)} \approx 0.2186$, and $x_{4}^{(24)} \approx 0.7814$. The rest point is unstable with respect to the edge. 
bioRxiv preprint doi: https://doi.org/10.1101/2021.08.05.455237; this version posted August 6, 2021. The copyright holder for this preprint (which was not certified by peer review) is the author/funder, who has granted bioRxiv a license to display the preprint in perpetuity. It is made available under aCC-BY-NC-ND 4.0 International license.

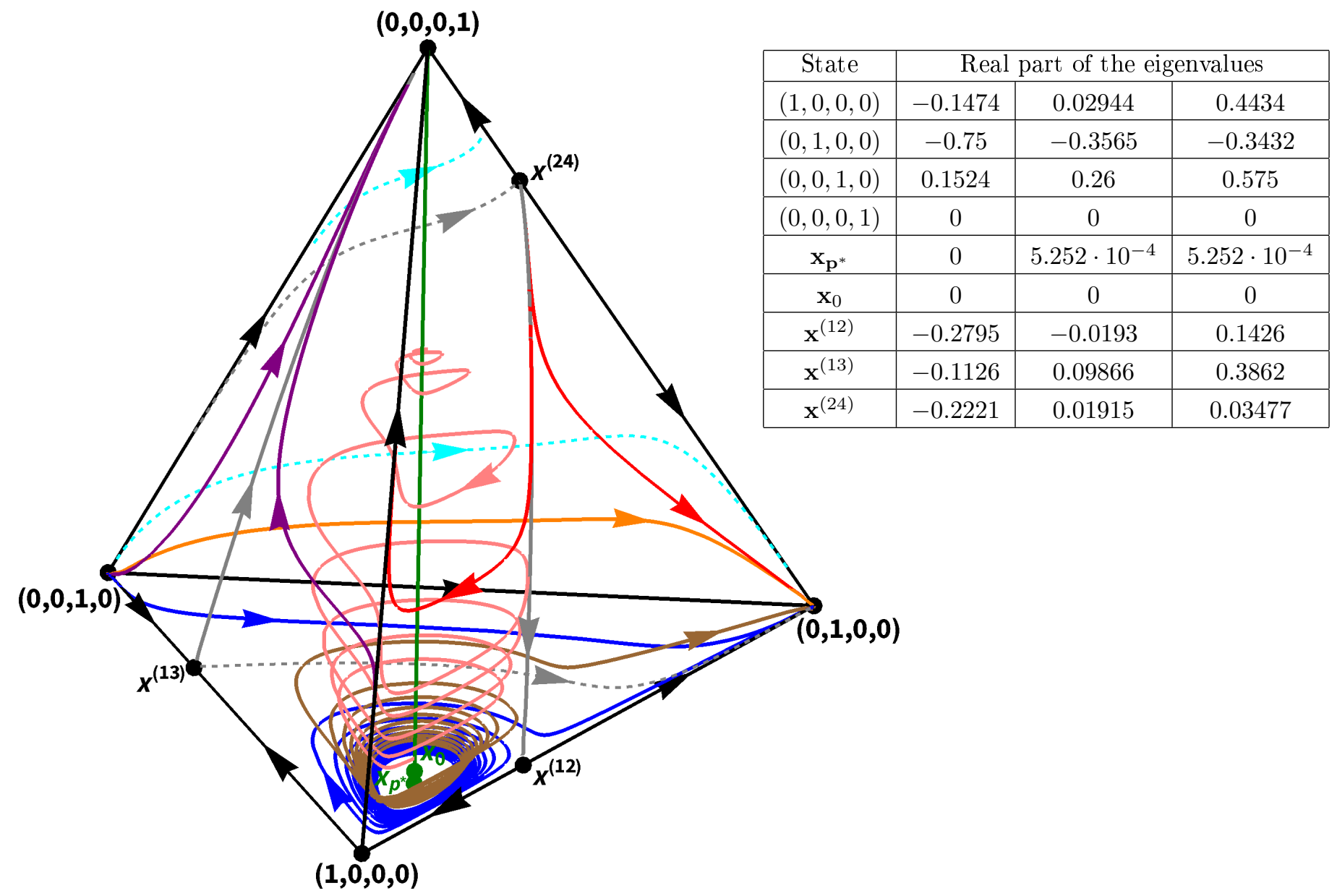

Figure 6. The phase portrait of the replicator dynamics with respect to phenotypes $\mathbf{e}_{1}, \mathbf{e}_{2}, \mathbf{e}_{3}$ and $\mathbf{p}^{*}=(1 / 3,1 / 3,1 / 3)$. The payoff matrix $\mathrm{A}$ and the time constraint matrix $\mathrm{T}$ are given in $(3.14) . \mathbf{x}_{\mathbf{p}^{*}}=\left(x_{\mathbf{p}^{*}, 1}, x_{\mathbf{p}^{*}, 2}, x_{\mathbf{p}^{*}, 3}, x_{\mathbf{p}^{*}, 4}\right)$ is the state corresponding to strategy $\mathbf{p}^{*}=(1 / 3,1 / 3,1 / 3)$ through Lemma 2.2 on the face determined by the vertices $(1,0,0,0)$, $(0,1,0,0)$ and $(0,0,1,0): x_{\mathbf{p}^{*}, 1} \approx 0.772, x_{\mathbf{p}^{*}, 2} \approx 0.147, x_{\mathbf{p}^{*} 3}=1-x_{\mathbf{p}^{*}, 1}-x_{\mathbf{p}^{*}, 2}, x_{\mathbf{p}^{*} 4}=0$. It agrees with $\mathbf{x}\left(\mathbf{p}^{*}\right)$ in Figure 5 . Every state on the green segment between $(0,0,0,1)$ and $\mathbf{x}_{\mathbf{p}^{*}}$ corresponds to strategy $\mathbf{p}^{*}$ through Lemma 2.2 . Hence every point of the segment is a rest point of the replicator dynamics. One of the three eigenvalues of the linearization of the replicator dynamics (3.10) at these states is zero. At $\mathbf{x}_{0}$, all of the eigenvalues has zero real part. The states on the segment under $\mathbf{x}_{0}$ have two eigenvalues with positive real part, so they are all unstable though they correspond to the UESS $\mathbf{p}^{*}$. The states between $\mathbf{x}_{0}$ and $(0,0,0,1)$ whereas have two eigenvalues with negative real part. The state $(0,0,0,1)$ is stable (but not asymptotically). In the table, we give the real parts of the eigenvalues of the linearization of the replicator dynamics at the rest points indicated in the phase portrait.

It appears that there is a separatrix connecting the rest point $\mathbf{x}^{(24)}$ with the rest point $\mathbf{x}^{(12)}$ on the face determined by the vertices $(1,0,0,0),(0,1,0,0)$ and $(0,0,0,1)$. The separatrix splits the face into two parts. The (interior) orbits start from $\mathbf{x}^{(24)}$ and end in the stable (moreover asymptotically stable with respect to the face) rest point $(0,0,0,1)$ on the part falling toward the edge between $(1,0,0,0)$ and $(0,0,0,1)$ while in the asymptotically stable rest point $(0,1,0,0)$ on the other part of the face.

Also, it seems that a separatrix runs from $\mathbf{x}^{(13)}$ to $(0,0,0,1)$ on the face determined by the states $(1,0,0,0),(0,0,1,0)$ and $(0,0,0,1)$. The orbits in the interior of the face all end in the state $(0,0,0,1)$ but the orbits falling the $(1,0,0,0)$ side of the separatrix start from the state $(1,0,0,0)$ whereas those falling the $(0,0,1,0)$ side of the separatrix start from the state $(0,0,1,0)$.

It appears that there is also a separatrix on the face determined by the vertices $(0,1,0,0)$, $(0,0,1,0)$ and $(0,0,0,1)$ connecting the state $(0,0,1,0)$ to $\mathbf{x}^{(24)}$. The orbits in the interior of 
the face start from $(0,0,1,0)$. The orbits located on the $(0,1,0,0)$ side of the separatrix run into the state $(0,1,0,0)$ while those on the $(0,0,0,1)$ side of the separatrix end in the sate $(0,0,0,1)$.

In this case, state $\mathbf{x}_{0}$ is the sate $\mathbf{x}^{E}\left(t_{0} / 3, t_{0} / 3, t_{0} / 3,1-t_{0}\right)$ where

$$
t_{0}=\frac{61518486052+3148879999 \sqrt{469}}{131829933359} \approx 0.9839
$$

so its coordinates are $\left(x_{0}\right)_{1} \approx 0.7596,\left(x_{0}\right)_{2} \approx 0.1446,\left(x_{0}\right)_{3} \approx 0.07981$ and $\left(x_{0}\right)_{4}=1-t_{0} \approx$ 0.01607 .

\section{Conclusion}

At first glance, analyzing the relationship between models on monomorphic and polymorphic populations can primarily appear to be a mathematical problem. However, it is connected to the question of diversity in biology. In the theory of classical evolutionary matrix games, for instance, the existence of an interior monomorphic UESS implies stable diversity in polymorphic situations through strong stability (Cressman 1990, Cressman 1992). Therefore, to make clear the difference between the monomorphic and polymorphic models is an interesting question from the viewpoint of the biology, as well.

The examples demonstrates that the relationship between monomorphic and polymorphic evolutionary stability valid for the case of classical evolutionary matrix games does not work under time constraints in three or higher dimension. Namely, the usual static uniform uninvadability does not imply the asymptotic stability of the corresponding state of the replicator dynamics. The question arises whether it could be given a biological relevant static condition which is weaker than the strict Nash equilibrium condition but it ensures the stability of the corresponding state in the polymorphic case.

\section{Acknowledgement}

This research was supported by

- by NKFIH, Hungary KKP 129877 (to T. V.);

\section{References}

Akin E (1982) Exponential families and game dynamics. Can J Math 34:374-405

Bomze I M, Weibull J (1995) Does neutral stability imply Lyapunov stability? Games Econ Behav 11:173-192

Broom M, Rychtář J (2013) Game-theoretical models in biology. Mathematical and computational biology. Chapman \& Hall/CRC, New York

Cressman R (1990) Strong stability and density-dependent evolutionarily stable strategies. J Theor Biol 145:147-165

Cressman R (1992) The stability concept of evolutionary game theory. Berlin: Springer 
Cressman R, Garay J, Varga Z (2003) Evolutionarily stable sets in the single-locus frequencydependent model of natural selection. J Math Biol 47(5):465-482

Garay J, Csiszár V, Móri TF (2017) Evolutionary stability for matrix games under time constraints. J Theor Biol 415:1-12

Garay J, Cressman R, Móri TF, Varga T (2018) The ESS and replicator equation in matrix games under time constraints. J Math Biol 76:1951-1973

Hofbauer J, Schuster P and Sigmund K (1979) A note on evolutionary stable strategies and game dynamics. J Theor Biol 81:609-612

Hofbauer J and Sigmund K (1998) Evolutionary Games and Population Dynamics. Cambridge University Press

Kong Q (2014) A Short Course in Ordinary Differential Equations. Springer

Maynard Smith J (1982) Evolution and the Theory of Games. Cambridge University Press

Maynard Smith J and Price G (1973) The logic of animal conflicts. Nature 246:15-18

Pohley H J, Thomas B (1983) Non-linear ESS-models and frequency dependent selection. BioSystems 16:87-100

Rudin W (1953) Principles of Mathematical Analysis. McGraw-Hill (New York, Toronto, London)

Taylor P D, Jonker L B (1978) Evolutionary stable strategies and game dynamics. Math Biosci 40:145-156

Thomas B (1985) On evolutionarily stable sets. J Math Biol 22:105-115

Thomas G B (2014) Thomas' Calculus. Pearson

Varga T, Móri TF, Garay J (2020) The ESS for evolutionary matrix games under time constraints and its relationship with the asymptotically stable rest point of the replicator dynamics. J Math Biol 80:743-774

Zeeman EC (1980) Population dynamics from game theory. In: Global theory of dynamical systems. Lecture Notes in Mathematics 819. New York: Springer-Verlag

Zeeman EC (1981) Dynamics of evolution of animal conflicts. J Theor Biol 89:249-270 